\title{
Meridional Atmospheric Heat Transport Constrained by Energetics and Mediated by Large-Scale Diffusion
}

\author{
KYLE C. ARMOUR \\ School of Oceanography and Department of Atmospheric Sciences, University of Washington, Seattle, Washington \\ NiCHOLAS SiLER \\ College of Earth, Ocean, and Atmospheric Sciences, Oregon State University, Corvallis, Oregon \\ AARON DONOHOE \\ Applied Physics Laboratory, University of Washington, Seattle, Washington \\ GERARD H. ROE \\ Department of Earth and Space Sciences, University of Washington, Seattle, Washington
}

(Manuscript received 29 August 2018, in final form 19 March 2019)

\begin{abstract}
Meridional atmospheric heat transport (AHT) has been investigated through three broad perspectives: a dynamic perspective, linking AHT to the poleward flux of moist static energy (MSE) by atmospheric motions; an energetic perspective, linking AHT to energy input to the atmosphere by top-of-atmosphere radiation and surface heat fluxes; and a diffusive perspective, representing AHT in terms downgradient energy transport. It is shown here that the three perspectives provide complementary diagnostics of meridional AHT and its changes under greenhouse gas forcing. When combined, the energetic and diffusive perspectives offer prognostic insights: anomalous AHT is constrained to satisfy the net energetic demands of radiative forcing, radiative feedbacks, and ocean heat uptake; in turn, the meridional pattern of warming must adjust to produce those AHT changes, and does so approximately according to diffusion of anomalous MSE. The relationship between temperature and MSE exerts strong constraints on the warming pattern, favoring polar amplification. These conclusions are supported by use of a diffusive moist energy balance model (EBM) that accurately predicts zonal-mean warming and AHT changes within comprehensive general circulation models (GCMs). A dry diffusive EBM predicts similar AHT changes in order to satisfy the same energetic constraints, but does so through tropically amplified warming - at odds with the GCMs' polar-amplified warming pattern. The results suggest that polar-amplified warming is a near-inevitable consequence of a moist, diffusive atmosphere's response to greenhouse gas forcing. In this view, atmospheric circulations must act to satisfy net AHT as constrained by energetics.
\end{abstract}

\section{Introduction}

Large-scale atmospheric motions predominantly act to transport energy poleward-from the warm and moist tropics, where insolation is strong, to cold and dry polar regions, where insolation is weak (e.g., Trenberth and Caron 2001; Trenberth and Stepaniak 2003; Fasullo and Trenberth 2008; Donohoe and Battisti 2012). As a consequence of meridional atmospheric heat transport (AHT), Earth's climate is more temperate than it would

Corresponding author: Kyle C. Armour, karmour@uw.edu otherwise be, exhibiting a weaker pole-to-equator temperature gradient (e.g., Hartmann 2016). Under greenhouse gas forcing, changes in AHT play a primary role in shaping the pattern of climate change, such as the degree of polar amplification (Hwang et al. 2011; Alexeev and Jackson 2013; Feldl and Roe 2013a; Rose et al. 2014; Pithan and Mauritsen 2014; Roe et al. 2015; Merlis and Henry 2018; Bonan et al. 2018; Stuecker et al. 2018) and the meridional pattern of hydrologic cycle changes (e.g., Held and Soden 2006; Siler et al.2018). A key question is this: What processes govern meridional AHT and its changes? 
Here we compare three complementary perspectivesdynamic, energetic, and diffusive - on meridional AHT. We first consider each perspective in the context of climatological AHT as derived from atmospheric reanalyses and satellite observations. We then consider each perspective in the context of AHT changes under greenhouse gas forcing as simulated by comprehensive global climate models (GCMs). Finally, we seek to reconcile the perspectives within a moist energy balance model framework. We show that, together, energetic and diffusive perspectives provide fundamental insights into how meridional AHT is constrained to change under climate forcing and how those changes shape the pattern of surface warming.

\section{Three perspectives on meridional heat transport}

\section{a. A dynamic perspective}

A traditional description of meridional AHT is in terms of dynamical processes. From this perspective, AHT arises from the poleward flux of moist static energy (MSE) by the dominant atmospheric motions. In the tropics, meridional energy transport is primarily accomplished by the mean meridional circulation (MMC) associated with the Hadley cell. Total energy transport in the Hadley cell is a small residual of offsetting contributions from its lower (equatorward) and upper (poleward) branches: moist, warm air is drawn equatorward near the surface and dry air is returned aloft, but because MSE (including potential energy) increases slightly with height in the tropical atmosphere, ${ }^{1}$

$$
[\overline{m v}]=\underbrace{[\bar{m}][\bar{v}]}_{\text {MMC }}+\underbrace{\overline{[m]^{\prime}[v]^{\prime}}}_{\text {TOC }}+\underbrace{\left[\bar{m}^{*} \bar{v}^{*}\right]}_{\text {stationary eddies }}+\underbrace{\left[\overline{m^{*^{\prime}} v^{*^{\prime}}}\right]}_{\text {transient eddies }},
$$

where primes denote deviations from the time mean and asterisks denote deviations from the zonal mean; TOC denotes the transient overturning circulation, which is small everywhere in the annual mean.

From the dynamic perspective, meridional AHT arises from energy transport associated with distinct atmospheric circulations at different latitudes, each showing pronounced and abrupt variations in meridional structure in AHT (Fig. 1a). Remarkably, when AHT associated with each circulation component is summed together they blend "seamlessly" to produce a net AHT without pronounced and abrupt meridional variations

\footnotetext{
${ }^{1}$ Except through the local minimum in the lower troposphere.
}

energy is larger in the upper branch, resulting in poleward energy transport overall.

Outside of the tropics, meridional energy transport is primarily accomplished by eddies, which advect moist, tropical air poleward while simultaneously drawing cool, dry air equatorward from high latitudes. The poleward energy transport from transient eddies dominates over that of stationary eddies in the annual mean, while the MMC associated with the Ferrel cells result in modest equatorward energy transport in midlatitudes.

We derive annual-mean meridional AHT from 6-hourly meridional velocity $(v)$ and MSE (denoted by $\left.m=c_{p} T+L_{v} q+g z\right)$ of air from the ECMWF interim reanalysis (ERA-Interim) (appendix A; Dee et al. 2011), where $T$ is temperature, $c_{p}$ is specific heat of air at constant pressure, $L_{v}$ is latent heat of vaporization, $q$ is specific humidity, and $g z$ is potential energy at height $z$ above the surface. We diagnose climatological northward AHT, denoted by $F(x)$, where $x$ is the sine of latitude, according to

$$
F(x)=\frac{2 \pi a}{g}\left(1-x^{2}\right)^{1 / 2} \int \overline{[m v]} d p,
$$

where $a$ is the radius of Earth, $g$ is acceleration due to gravity, $\left(1-x^{2}\right)^{1 / 2}$ accounts for spherical geometry, and the integral over pressure $p$ is from the top of the atmosphere (TOA) to the surface; overbars denote time means and square brackets denote zonal means. AHT can further be partitioned into distinct atmospheric circulations (e.g., Holton and Hakim 2013):

(Trenberth and Stepaniak 2003). Net AHT has a peak magnitude of about $4 \mathrm{PW}$ at around $40^{\circ}$ latitude in both hemispheres and is poleward everywhere except in the deep tropics where energy is transported southward across the equator (Fig. 1a).

The dynamic perspective on meridional AHT is appealing for its explicit connection to the general atmospheric circulation. However, we lack a theory for how circulation components that vary so greatly with latitude conspire to produce such seamless meridional structure and hemispheric symmetry in net AHT (Trenberth and Stepaniak 2003). Moreover, while the dynamic perspective permits a diagnostic partitioning of AHT into components associated with distinct atmospheric motions, it does not, by itself, constrain the net AHT to which they sum. 

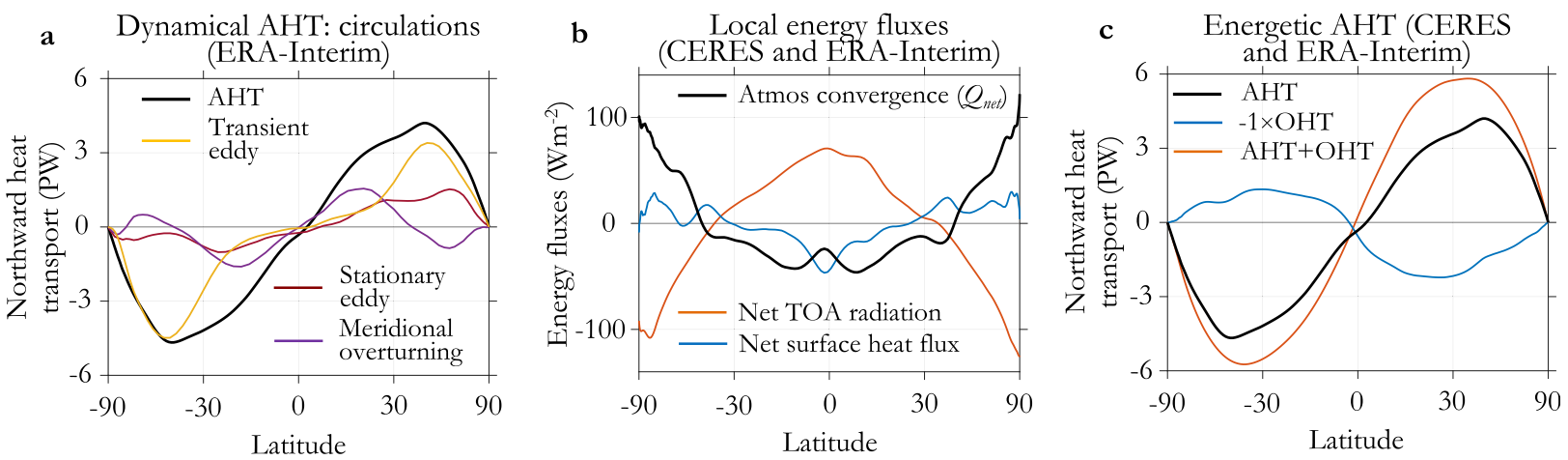

d Moist vs dry partitioning of AHT
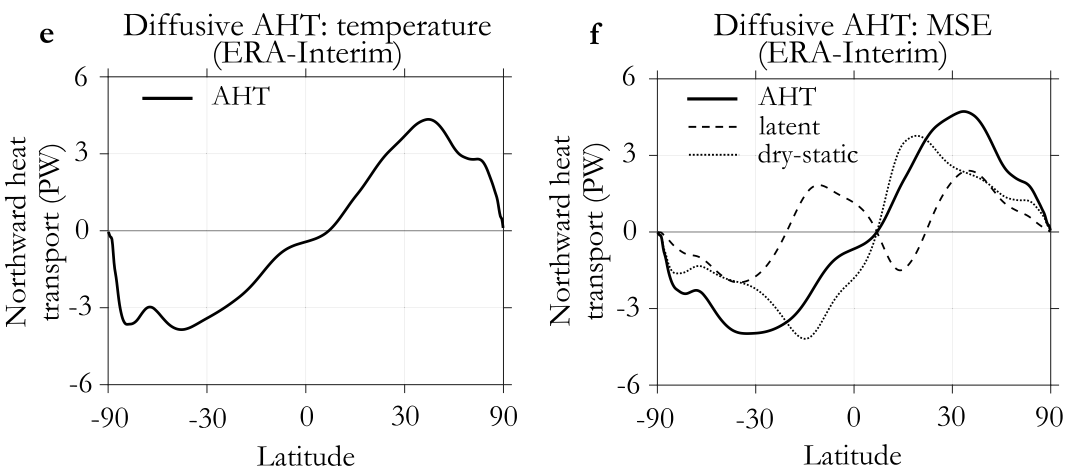

FIG. 1. Dynamic, energetic, and diffusive perspectives on climatological meridional atmospheric heat transport (AHT) (CERES satellite observations and ERA-Interim data). (a) Northward AHT partitioned into atmospheric circulation components-transient eddy, stationary eddy, and meridional overturning (mean and transient meridional overturning combined) - calculated from ERA-Interim according to Eqs. (1) and (2). (b) Zonal-mean energy fluxes into the atmospheric column derived from CERES and ERA-Interim, partitioned into net TOA radiation, surface heat fluxes, and atmospheric heat flux convergence. (c) AHT derived by meridionally integrating zonal-mean energy fluxes according to Eq. (4); red line shows AHT implied by net TOA radiation; blue line shows AHT implied by surface heat fluxes [opposite sign of ocean heat transport, (OHT)]; black line shows net AHT implied as the sum of the others. (d) AHT partitioned into latent energy and dry-static energy components, calculated from ERA-Interim according to Eq. (1). (e) AHT derived from diffusion of temperature [Eq. (5)] applied to near-surface air temperature from ERA-Interim. (f) AHT derived from diffusion of MSE [Eq. (6)] applied to near-surface air MSE from ERA-Interim, with Hadley cell extension to partition AHT into latent energy and dry-static energy components in the tropics (appendix B).

\section{b. An energetic perspective}

A second perspective is that meridional AHT is as it needs to be to meet the net energetic demands of TOA radiation and surface energy fluxes. Because absorbed shortwave radiation exceeds outgoing longwave radiation at low latitudes, while outgoing longwave exceeds absorbed shortwave at high latitudes, total planetary heat transport must act to diverge energy from the tropics and converge energy in polar regions to maintain local energy balance (e.g., Hartmann 2016). Only a small portion of this energetic demand is met by meridional ocean heat transport (OHT), leaving most of the energy transport to be accomplished by the atmosphere.

In this view, the zonal-mean net heating of the atmosphere, $Q_{\text {net }}$, must be balanced, on long time scales, by the divergence of northward AHT:

$$
Q_{\text {net }}(x)=\frac{1}{2 \pi a^{2}} \frac{d F}{d x} .
$$

In turn, northward AHT can be calculated from the meridional integral of $Q_{\text {net }}(x)$ :

$$
F(x)=2 \pi a^{2} \int_{-1}^{x} Q_{\text {net }}(\tilde{x}) d \tilde{x} .
$$

We derive $Q_{\text {net }}$ from net TOA radiation observed from the Clouds and the Earth's Radiant Energy System Energy Balance and Filled product (CERES EBAF; Loeb et al. 2009) combined with net surface heat fluxes from ERA-Interim (Fig. 1b; appendix A). The result, shown in Fig. 1c, is AHT with peak magnitude of about 4 PW at around $40^{\circ}$ latitude in both hemispheres and seamless meridional structure.

Net meridional AHT diagnosed from the atmospheric energy budget (Fig. 1c) agrees with that diagnosed from atmospheric circulations (Fig. 1a), as it must (appendix A). However, the energetic perspective links AHT to a different set of climate processes. Meridionally 
integrating the individual components of $Q_{\text {net }}$ (Fig. 1b) according to Eq. $(4)^{2}$ shows that the meridional structure of meridional AHT largely mirrors that required by TOA radiation and is partially compensated by surface heat fluxes, which reflect OHT (Fig. 1c). From the energetic perspective, seamless meridional structure and hemispheric symmetry of AHT arise because net TOA radiation varies seamlessly with latitude and is nearly symmetric between the hemispheres (Fig. 1b; Voigt et al. 2013; Stephens et al. 2015).

While the energetic perspective does not require knowledge of the specific atmospheric motions by which AHT is accomplished, it postulates that those motions must collectively satisfy net energetic constraints. Stone (1978) pioneered this reasoning by arguing that total planetary heat transport (AHT + OHT) is determined by the meridional structure of absorbed solar radiation, independent of the dynamical details of the oceanatmosphere system; this approximation holds to the degree that outgoing longwave radiation is insensitive to variations in surface temperature. An implication is that for fixed TOA radiation, AHT must adjust to any change in OHT to maintain local energy balance-a compensation originally proposed by Bjerknes (1964). Imperfect compensation arises only to the degree that TOA radiation responds to changes in surface temperature (Rose and Ferreira 2013; Liu et al. 2016). More recently, Donohoe and Battisti (2012) used energetic arguments to link AHT biases to cloud biases in GCMs based on a strong correlation between AHT and poleto-equator gradients in absorbed solar radiation across models. The spatial pattern of absorbed solar radiation is also thought to govern climatological poleward AHT across different climate states, such as those simulated by varying geometrical constraints on ocean circulation (Enderton and Marshall 2009) or varying Earth's rotation rate (Liu et al. 2017).

The energetic perspective also provides a framework for understanding the latitudinal position of the intertropical convergence zone (ITCZ): annual-mean ascent north of the equator permits net MSE to be transported southward across the equator in the upper branch of the Hadley cell, as required to balance stronger heating of the Northern Hemisphere atmosphere (Kang et al. 2008; Frierson and Hwang 2012; Hwang and Frierson 2013; Donohoe et al. 2013, 2014). An implication is that the peak in zonal-mean rainfall resides north of the equator

\footnotetext{
${ }^{2}$ The individual components of $Q_{\text {net }}(x)$ have nonzero globalmean values that we subtract (meridionally uniformily) from the integrand of Eq. (4) to ensure that $F(x)$ implied by each component goes to zero at the poles.
}

in the annual mean due to hemispheric asymmetry of high-latitude surface heat fluxes which, in turn, reflects northward OHT across the equator due to meridional overturning in the Atlantic Ocean (Figs. 1b,c; Frierson et al. 2013; Marshall et al. 2014).

The energetic perspective permits meridional AHT to be diagnosed from TOA radiation and surface energy fluxes without knowledge of atmospheric circulations. It further links the seamless meridional structure and symmetry of AHT to that of net TOA radiation. However, it is unclear to what extent the energetic perspective can be thought of as a constraint on meridional AHT given that TOA radiation depends (at least weakly) on the patterns of atmospheric and surface temperatures, which, in turn, depend on AHT.

\section{c. A diffusive perspective}

A third perspective comes from the representation of AHT as a macroturbulent (Held 1999) or diffusive process. The traditional assumption (e.g., Budyko 1969; Sellers 1969; Stone 1978; North 1975, 1981; Merlis 2014; Wagner and Eisenman 2015) is that AHT is proportional to the meridional gradient in zonal-mean nearsurface air temperature $T(x)$, which on a sphere gives

$$
F(x)=-\frac{2 \pi p_{s}}{g} c_{p} D_{d}\left(1-x^{2}\right) \frac{d T}{d x}
$$

where $D_{d}$ is a constant "dry" diffusion coefficient with units of $\mathrm{m}^{2} \mathrm{~s}^{-1}$ and $p_{s}$ is surface air pressure $(1000 \mathrm{hPa})$. More recent studies (e.g., Flannery 1984; Frierson et al. 2007; Hwang and Frierson 2010; Hwang et al. 2011; Rose et al. 2014; Roe et al. 2015; Liu et al. 2016; Merlis and Henry 2018; Siler et al. 2018; Bonan et al. 2018) account for latent heat by assuming that AHT is proportional to the meridional gradient in zonal-mean near-surface MSE, denoted by $h(x)=c_{p} T(x)+L_{v} q(x)$, where $q(x)$ is near-surface specific humidity, giving

$$
F(x)=-\frac{2 \pi p_{s}}{g} D_{m}\left(1-x^{2}\right) \frac{d h}{d x},
$$

where $D_{m}$ is a constant "moist" diffusion coefficient with units of $\mathrm{m}^{2} \mathrm{~s}^{-1}$.

We derive $F(x)$, shown in Figs. 1e and 1f, using zonalmean $T(x)$ and $h(x)$ from ERA-Interim (Fig. 2; appendix A). Following Hwang and Frierson (2010), we approximate near-surface MSE assuming a flat surface (constant $p_{s}$ ) and fixed (80\%) relative humidity; $q(x)$ is governed by the Clausius-Clapeyron relation and depends only on $T(x)$. Without a priori knowledge of the effective dry or moist diffusivities of the atmosphere, we choose values $\left(D_{d}=2.2 \times 10^{6} \mathrm{~m}^{2} \mathrm{~s}^{-1}\right.$ and $D_{m}=0.96 \times$ $10^{6} \mathrm{~m}^{2} \mathrm{~s}^{-1}$ ) that minimize the mean square error 
Climatological T and MSE (ERA-Interim)

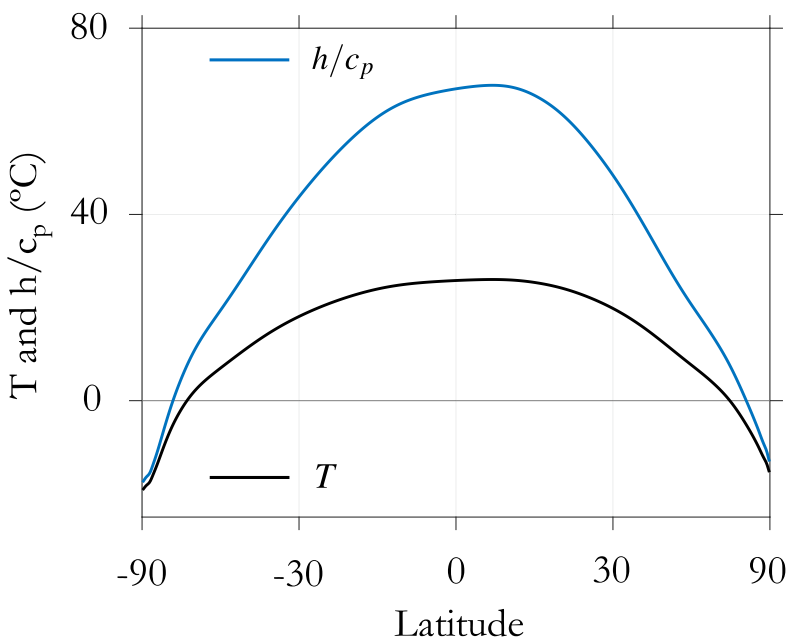

FIG. 2. Climatological zonal- and annual-mean, near-surface air temperature, and moist static energy (ERA-Interim). Near-surface air temperature (black line) and MSE (divided by $c_{p}$; blue line).

between AHT calculated by Eqs. (5) and (6) and that calculated from either Eq. (1) or Eq. (4) (Figs. 1a,c). The value of $D_{m}$ is within $10 \%$ of that diagnosed from the climatology of GCMs by Hwang and Frierson (2010). Importantly, $D_{m}$ and $D_{d}$ are independent of latitude. The factor of 2 difference between $D_{m}$ and $D_{d}$ reflects the pole-to-equator gradient of $h(x) / c_{p}$ being approximately twice as large as that of $T(x)$ due to the nearly exponential increase in $q(x)$ with temperature (Fig. 2; Flannery 1984; Merlis and Henry 2018).

Equations (5) and (6) do not reproduce all features of meridional AHT as calculated from atmospheric circulations or the atmospheric energy budget (cf. Figs. 1e,f with Figs. 1a,c). This is unsurprising, given 1) the strong idealization that diffusivity is independent of latitude and acts on gradients of near-surface temperature or MSE and 2) the intuition that atmospheric motions should behave diffusively, in some approximate sense, only in the extratropical atmosphere where transient eddies stir temperature and moisture efficiently (Held 1999; see Fig. 1a herein). Yet, meridional AHT derived from this simple principle of diffusive, downgradient energy transport broadly captures the meridional structure of AHT and its peak magnitude of about $4 \mathrm{PW}$ at around $40^{\circ}$ latitude in both hemispheres (Figs. 1e,f). Despite its great dynamical complexity, the overall tendency of the atmosphere appears to be that of downgradient energy transport from the warm, moist tropics to the cold, dry polar regions.

From the diffusive perspective, seamless meridional structure and hemispheric symmetry of AHT arise from the seamless meridional variation and approximate hemispheric symmetry of $T(x)$ or $h(x)$. AHT is poleward everywhere except in the deep tropics where southward energy transport across the equator arises from the maximum in $T(x)$ or $h(x)$ residing north of the equator in the annual mean (Fig. 2).

The diffusive perspective complements Stone's (1978) energetic reasoning regarding constraints on total planetary heat transport (AHT + OHT): provided that atmospheric circulations act to transport energy downgradient in a sufficiently diffusive manner, AHT will readily adjust to changes in TOA radiation or surface heat fluxes independent of the dynamical details of the system; Bjerknes compensation of OHT changes can be understood as atmospheric energy divergence adjusting more than TOA radiation does in response to changes in surface temperature (Liu et al. 2016).

The diffusive perspective links the meridional structure of AHT directly to that of near-surface air temperature or MSE; yet, $T(x)$ and $h(x)$ are themselves influenced by AHT. Moreover, it is unclear why a diffusive approximation works well in the deep tropics where transient eddies contribute relatively little to AHT (Fig. 1a). That similar patterns of AHT can be obtained using different assumptions about the atmospheric energy budget- $T(x)$ in Eq. (5) or $h(x)$ Eq. (6) -is cause for rumination. In sections $3 c$ and 4 , we will consider which diffusive description, if either, is realistic.

An alternative partitioning of AHT is into the transport of energy associated with moisture (latent energy, $L_{v} q$ ) and temperature (dry-static energy, $\left.c_{p} T+g z\right)$. This partitioning can be calculated in either of two ways. From a dynamic perspective, we apply Eq. (1) separately to $c_{p} T+g z$ and $q$ fields from ERA-Interim to estimate meridional dry-static energy and latent energy transports, respectively. From an energetic perspective, we apply Eq. (4) to the zonal-mean latent heat flux convergence implied by net precipitation minus evaporation from ERAInterim; this gives an estimate of meridional latent energy transport, which we subtract from net AHT to estimate meridional dry-static energy transport. Both dynamic and energetic estimates give the same result, shown in Fig. 1d: the transport of dry-static energy is poleward at all latitudes, while the transport of latent energy is poleward outside of the tropics and equatorward in the vicinity of the Hadley cell. There is strong compensation between latent energy and dry-static energy transports in the tropics, but each contributes approximately equal poleward AHT in the midlatitudes (Trenberth and Stepaniak 2003). Despite pronounced meridional variations in the moist and dry components, 
they sum to produce a seamless meridional structure in net AHT.

Without a representation of moisture transport, temperature diffusion cannot replicate this partitioning of moist and dry AHT. Can MSE diffusion? In the extratropics, Eq. (6) applied to $c_{p} T(x)$ and $L_{v} q(x)$ separately reproduces the poleward transport of latent energy and dry-static energy with approximately equal partitioning (Fig. 1f). Within the tropics, a diffusive approximation cannot represent the observed upgradient advection of moisture. However, following Siler et al. (2018), we can extend the diffusive perspective to capture tropical moisture transport by implementing a minimal representation of the Hadley cell (appendix B) that partitions the net AHT [Eq. (6)] into Hadley cell and eddy components. In the tropics, the Hadley cell parameterization is active and dominates moisture transport, resulting in an upgradient flux of latent energy. In the extratropics, eddies dominate and latent energy is fluxed downgradient.

Diffusion of MSE (with Hadley cell extension) is able to capture the partitioning of latent energy and dry-static energy components of meridional AHT as set by distinct regimes of atmospheric motions (cf. Figs. 1d,f). Siler et al. (2018) explore the implications of diffusive moisture transport for the meridional structure of the hydrologic cycle and its changes under greenhouse gas forcing.

\section{IN SUM}

- The above perspectives-dynamic, energetic, and diffusive - are each widely used to diagnose meridional AHT. These perspectives are complementary given that they describe AHT from different levels of complexity and with distinct physical assumptions.

- From the observed climatology alone, it is not clear which perspective, if any, provides more fundamental insight into the processes governing meridional AHT. Importantly, all three perspectives are inherently diagnostic.

A strong demonstration of the merit of each perspective would be the ability to provide a mechanistic understanding of meridional AHT changes under climate forcing. We next consider the three perspectives in the context of global warming as simulated by comprehensive GCMs. While each perspective has been applied to GCM output previously (e.g., Hwang and Frierson 2010; Wu et al.2011; Huang and Zhang 2014), here we apply all three to the same set of CMIP5 simulations, permitting a novel comparison of the perspectives in a like-with-like way.

\section{Three perspectives on meridional heat transport changes under greenhouse gas forcing}

Driven by rising greenhouse gas concentrations, coupled GCMs robustly predict an increase in poleward AHT in the midlatitudes of both hemispheres (Fig. 3; Held and Soden 2006; Hwang and Frierson 2010; Zelinka and Hartmann 2012; Wu et al. 2011; Huang and Zhang 2014). Meanwhile, they predict little change or even a decrease in poleward AHT at high latitudes (Fig. 3; Hwang et al. 2011), even while producing polaramplified surface warming (Fig. 4a). What processes govern these AHT changes, and how are they connected to the meridional pattern of surface warming?

We analyze output from 11 GCMs participating in the most recent Coupled Model Intercomparison Project (CMIP5; Taylor et al. 2012). This subset of models reflects those that provide the necessary output for calculating AHT and its changes from all three perspectives (appendix C). For each GCM, we calculate anomalies in northward AHT, denoted by $F^{\prime}(x)$, as the difference between $F(x)$ averaged over a preindustrial control simulation and $F(x)$ at a century into a simulation of abrupt $\mathrm{CO}_{2}$ quadrupling (average over years 85115). We consider CMIP5-mean changes throughout. The use of large radiative forcing, model averaging, and averaging over 31 years at the centennial time scale allows us to study long-term, transient, forced changes. In section $4 \mathrm{c}$ we consider the response to $\mathrm{CO}_{2}$ forcing near equilibrium.

\section{a. A dynamic perspective}

A variety of atmospheric circulation changes have been found to occur in response to $\mathrm{CO}_{2}$ forcing: a narrowing and shifting of the ITCZ (e.g., Neelin et al. 2003; Huang et al. 2013; McFarlane and Frierson 2017); a slowdown and poleward expansion of the Hadley cell (e.g., Held and Soden 2006; Lu et al. 2007); poleward shifts of midlatitude jets and storm tracks (e.g., Yin 2005; Barnes and Polvani 2013; Mbengue and Schneider 2017, 2018); and changing planetary wave activity (e.g., Lee 2014; Liu and Barnes 2015; Graversen and Burtu 2016), among others. Each circulation change has the potential to modify meridional AHT. Yet, even in the absence of circulation changes, warming and moistening of the atmosphere would lead to AHT changes by modifying the MSE profiles on which climatological circulations act (e.g., Held and Soden 2006).

We use Eqs. (1) and (2) to diagnose $F^{\prime}(x)$ and its dynamical partitioning within the CMIP5 models (appendix C). The result is a robust increase in poleward AHT in the midlatitudes of both hemispheres (by about 0.3 PW at the climatological maxima) and a slight decrease 

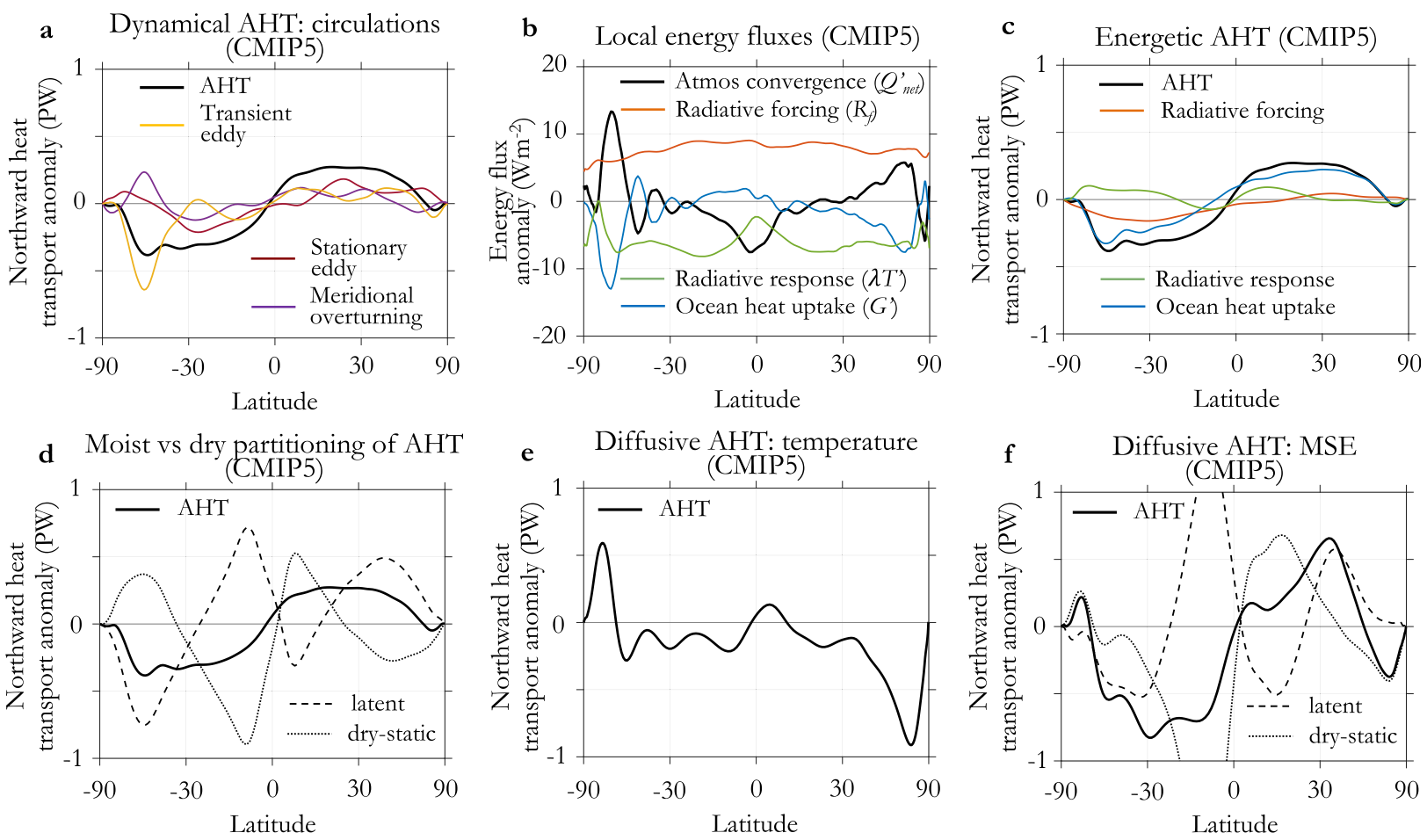

FIG. 3. Dynamic, energetic, and diffusive perspectives on meridional atmospheric heat transport changes (CMIP5-mean response at year 100 following abrupt $\mathrm{CO}_{2}$ quadrupling). (a) Anomalous northward AHT partitioned into atmospheric circulation components: transient eddy, stationary eddy, and meridional overturning (mean and transient meridional overturning combined). (b) Anomalous zonal-mean energy fluxes into the atmospheric column from radiative forcing, radiative response, surface heat fluxes (ocean heat uptake), and atmospheric heat flux convergence. (c) Anomalous AHT derived by meridionally integrating anomalous zonal-mean energy fluxes according to Eq. (11); red line shows anomalous AHT implied by radiative forcing; blue line shows anomalous AHT implied by ocean heat uptake; green line shows anomalous AHT implied by radiative response (feedbacks); black line shows net AHT implied as the sum of the others. (d) Anomalous AHT partitioned into latent energy and dry-static energy components. (e) Anomalous AHT derived from diffusion of temperature [Eq. (5)] applied to CMIP5 anomalous near-surface air temperature. (f) Anomalous AHT derived from diffusion of MSE [Eq. (6)] applied to CMIP5 anomalous near-surface MSE.

in poleward AHT into the Arctic (Fig. 3a), consistent with previous studies (e.g., Zelinka and Hartmann 2012; Huang and Zhang 2014). The relative contributions of the MMC, stationary eddies, and transient eddies to anomalous AHT vary with latitude. Yet, because of compensations between them, they sum to produce a net AHT anomaly that varies comparatively seamlessly with latitude.

The meridional structure of climatological AHT could be readily interpreted in terms of the dominant regimes of atmospheric circulation (section 2a; Fig. 1a). The meridional structure of AHT anomalies, however, does not obviously track expected changes in atmospheric circulations described above, nor does it reflect a simple enhancement of the climatological AHT associated with different circulation components (cf. Figs. 3a and 1a), except perhaps over the Southern Ocean. Moreover, it is unclear how AHT anomalies associated with distinct dynamical circulations at different latitudes are able to produce such a seamless structure in net
AHT changes, or why they would do so with approximate symmetry between hemispheres.

A reasonable conjecture is that energy transport by atmospheric motions must somehow adjust to satisfy some fundamental constraint on net AHT changes. We will argue that such a constraint arises naturally from the energetic perspective. Indeed, many recent studies explore causal links between changes in TOA radiation and atmospheric dynamics (Wu et al. 2011; Donohoe et al. 2013, 2014; Feldl et al. 2014; Ceppi et al. 2014; Voigt and Shaw 2015; Merlis 2015; Ceppi and Hartmann 2016; Voigt and Shaw 2016; Kay et al. 2016; Feldl and Bordoni 2016; McFarlane and Frierson 2017; WattMeyer and Frierson 2017; Ceppi and Shepherd 2017; Mbengue and Schneider 2017, 2018).

Partitioning net AHT changes into latent and drystatic energy contributions shows similar compensations across latitudes, with greater meridional variations in each component than in the total to which they sum (Fig. 3d). In the extratropics, poleward latent energy 
a Anomalous T and MSE (CMIP5)

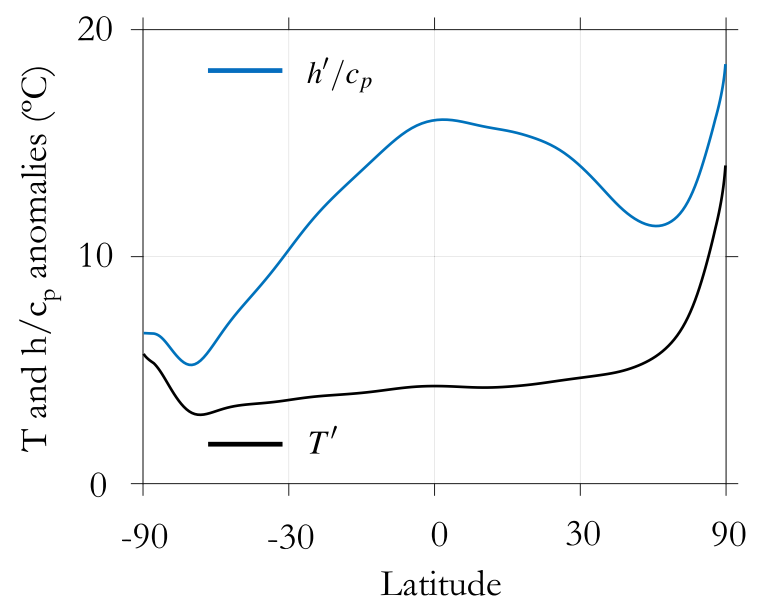

b Anomalous T and MSE (Moist EBM)

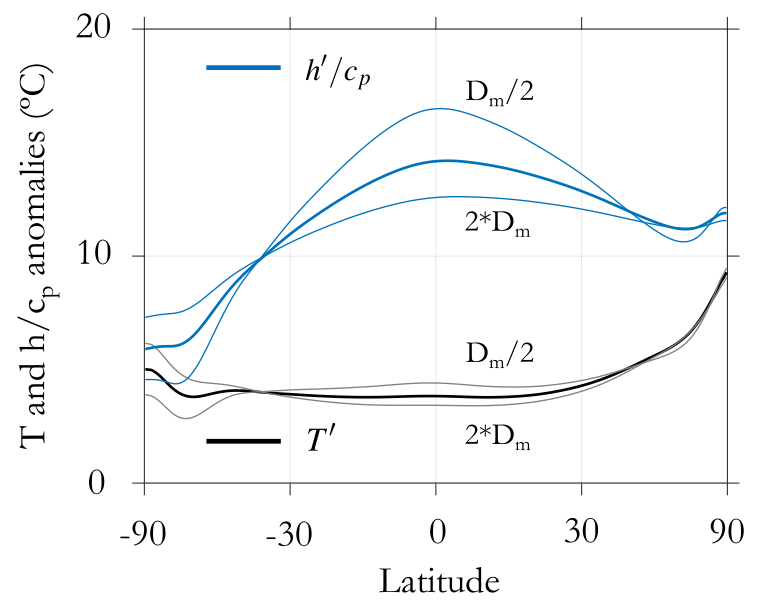

c Anomalous T (Dry EBM)

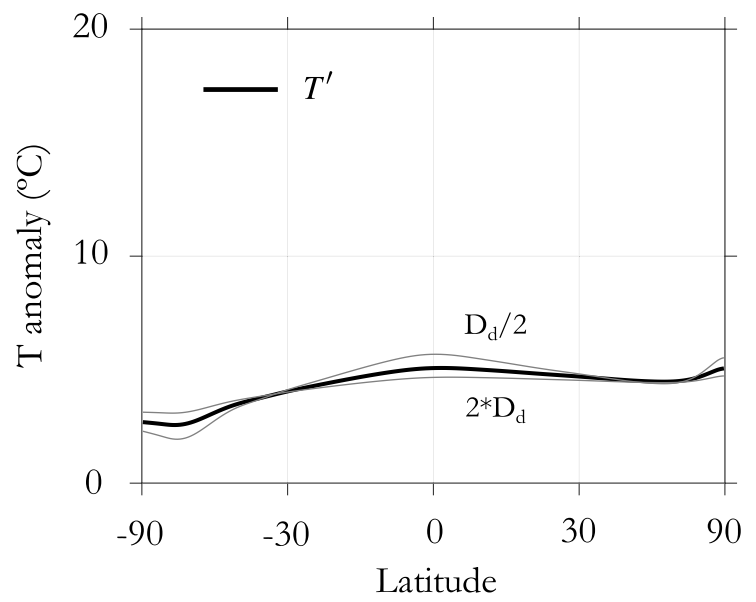

FIG. 4. Zonal- and annual-mean, near-surface air temperature, and moist static energy anomalies (CMIP5-mean, Moist EBM, and Dry EBM responses at year 100 following abrupt $\mathrm{CO}_{2}$ quadrupling). (a) CMIP5-mean anomalous near-surface air temperature transport increases while dry-static energy transport decreases; in the tropics, both equatorward latent energy transport and poleward dry-static energy transport increase. We will argue that this moist-dry partitioning of meridional AHT changes can be understood from the diffusive perspective (section 3c).

\section{b. An energetic perspective}

Changes in meridional AHT can be interpreted in terms of energetic constraints: in response to anomalous zonal-mean net heating of the atmosphere, $Q_{\text {net }}^{\prime}$, local energy balance must be regained through anomalous energy divergence:

$$
Q_{\mathrm{net}}^{\prime}(x)=\frac{1}{2 \pi a^{2}} \frac{d F^{\prime}}{d x}
$$

giving anomalous northward AHT in terms of the meridional integral of $Q_{\mathrm{net}}^{\prime}$ :

$$
F^{\prime}(x)=2 \pi a^{2} \int_{-1}^{x} Q_{\mathrm{net}}^{\prime}(\tilde{x}) d \tilde{x} .
$$

A useful partitioning of TOA radiation changes is into radiative forcing, denoted by $R_{f}(x)$, and radiative response to surface warming, denoted by $\lambda(x) T^{\prime}(x)$, where net radiative feedback $\lambda(x)$ (units of $\mathrm{W} \mathrm{m}^{-2} \mathrm{~K}^{-1}$ ) represents a linearization of zonal-mean radiative response with respect to zonal-mean surface temperature change $T^{\prime}(x)$ (e.g., Armour et al. 2013; Feldl and Roe 2013b; Rose et al. 2014; Roe et al. 2015). This gives

$$
Q_{\mathrm{net}}^{\prime}(x)=\lambda(x) T^{\prime}(x)+R_{f}(x)+G^{\prime}(x),
$$

where $G^{\prime}(x)$ is the change in net upward surface heat flux (with negative values reflecting ocean heat uptake). CMIP5-mean patterns of $T^{\prime}(x), \lambda(x), R_{f}(x)$, and $G^{\prime}(x)$ are shown in Figs. 4a and 5 (see appendix $C$ for details of their calculation). The CMIP5-mean pattern of $\lambda(x)$ $T^{\prime}(x)$ is shown in Fig. 3b.

We diagnose changes in poleward AHT within the CMIP5 models using Eqs. (8) and (9). The result is increased poleward AHT in the midlatitudes of both hemispheres (by about $0.3 \mathrm{PW}$ at the climatological

(black line) and MSE (divided by $c_{p}$; blue line). (b) Moist EBM anomalous near-surface air temperature (black line) and MSE (divided by $c_{p}$; thick blue line). (c) Dry EBM anomalous nearsurface air temperature (thick black line). Thick lines in (b) and (c) show response with diffusivities derived from the ERA-Interim climatology, while thin lines show the results with diffusivities half or double those values (section 4). 


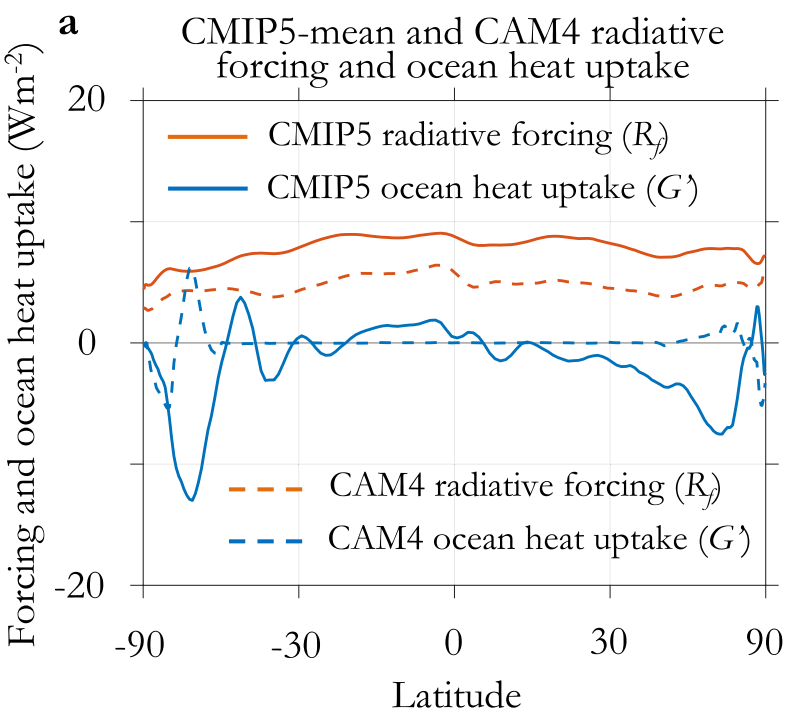

b

CMIP5-mean and CAM4 net radiative feedback

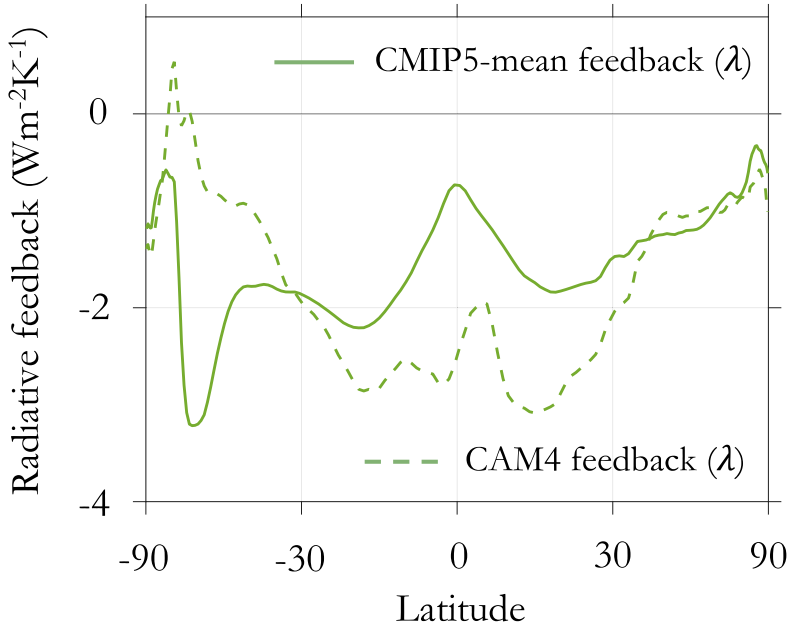

FIG. 5. Zonal-mean radiative forcing, ocean heat uptake, and radiative feedback (CMIP5 and CAM4). (a) CMIP5-mean radiative forcing under $\mathrm{CO}_{2}$ quadrupling (solid red line) and CAM4 radiative forcing under $\mathrm{CO}_{2}$ doubling (dashed red line); CMIP5mean ocean heat uptake at year 100 of $\mathrm{CO}_{2}$ quadrupling (solid blue line) and CAM4 ocean heat uptake under $\mathrm{CO}_{2}$ doubling at equilibrium (dashed blue line). (b) CMIP5-mean net radiative feedback at year 100 of $\mathrm{CO}_{2}$ quadrupling (solid green line) and CAM4 radiative feedback under $\mathrm{CO}_{2}$ doubling at equilibrium (dashed green line).

maxima) and slightly decreased poleward AHT into the Arctic (Fig. 3c). This agrees with AHT derived from the dynamic perspective (Fig. 3a), as it must (appendix C). However, from the energetic perspective, the increase in poleward AHT in midlatitudes is a consequence of increased energy input into the tropical atmosphere by $R_{f}(x)$ and $G^{\prime}(x)$, which is only weakly damped by the radiative response to warming (Fig. 3b). That is, in the tropics where the magnitude of $\lambda(x)$ is relatively small (reflecting a weak radiative response per degree of warming), restoring local energy balance requires anomalous atmospheric energy divergence and thus increased poleward AHT in the midlatitudes (Fig. 3c). Anomalous atmospheric energy convergence in the midlatitudes is balanced by an efficient radiative response to warming [more negative values of $\lambda(x)$ ] and by ocean heat uptake (Fig. 3b).

Changes in poleward AHT into the Arctic can be understood from the energetic perspective as well: despite large $T^{\prime}(x)$ (Fig. 4a), the TOA radiative response is relatively weak due to small (less negative) $\lambda(x)$; a decrease in poleward AHT (Fig. 3c) is thus required to balance anomalous energy input to the Arctic atmosphere from both $R_{f}(x)$ and $G^{\prime}(x)$ (Fig. 3b; Hwang et al. 2011).

Applying Eq. (8) to each component of $Q_{\text {net }}^{\prime}$ separately permits quantification of the poleward AHT changes implied by $\lambda(x) T^{\prime}(x), R_{f}(x)$, and $G^{\prime}(x)$ (Zelinka and Hartmann 2012; Huang and Zhang 2014). In this view, processes that preferentially add energy to the tropical atmosphere (e.g., $\mathrm{CO}_{2}$ forcing and water vapor feedback) or remove energy from the extratropical atmosphere (e.g., subpolar ocean heat uptake) act to increase poleward AHT in midlatitudes. Processes that preferentially remove energy from the tropical atmosphere (e.g., lapse-rate feedback) or add energy to the extratropical latitude atmosphere (e.g., lapse-rate and ice-albedo feedbacks) act to decrease poleward AHT in midlatitudes.

From the energetic perspective, poleward AHT changes most closely mirror those terms in $Q_{\text {net }}^{\prime}$ that show the greatest large-scale meridional structure. For the CMIP5 models, the radiative response to warming, $\lambda(x) T^{\prime}(x)$, varies little with latitude owing to small values of $\lambda(x)$ compensating large $T^{\prime}(x)$ at high latitudes (Fig. 3b); the pattern of radiative response thus implies little change in meridional AHT (Fig. 3c). Also, $R_{f}(x)$ varies relatively little with latitude (Fig. 3b), implying slightly increased poleward AHT in midlatitudes (Fig. 3c). The greatest meridional variations in $Q_{\text {net }}^{\prime}$ come from $G^{\prime}(x)$ (Fig. 3b). The meridional structure of AHT thus largely mirrors that implied by ocean heat uptake (Fig. 3c), consistent with the results of Huang and Zhang (2014) and with the large atmospheric heat flux convergence over the subpolar oceans (Fig. 3b) where sea surface warming is delayed by ocean circulations (Marshall et al. 2015; Armour et al. 2016). Results for individual models can be seen in Fig. $\mathrm{C} 1$ in appendix C.

The energetic perspective provides a powerful description of AHT changes in terms of the meridional 
patterns of radiative forcing, radiative feedbacks, and ocean heat uptake. Where the atmosphere is inefficient at radiating additional energy to space with warming (deep tropics and polar regions), local energy balance must be regained primarily through anomalous energy divergence; where the atmosphere is efficient at radiating additional energy to space with warming (midlatitudes), local energy balance can be regained, in part, through radiative response (Feldl and Roe 2013a; Roe et al. 2015). Yet, most of the structure in AHT arises from ocean heat uptake: where the oceans preferentially take up heat (subpolar oceans), the atmosphere must converge energy to maintain local energy balance. In this view, the approximate hemispheric symmetry in AHT changes arise from the approximate symmetry in Southern Ocean and North Atlantic Ocean heat uptake. This description is inherently diagnostic, however, given that the radiative response depends, at least weakly, on the pattern of surface warming, which, in turn, depends on meridional AHT changes.

\section{c. A diffusive perspective}

In section $2 \mathrm{c}$ we found that the principle of diffusive, downgradient energy transport produced reasonable representations of climatological meridional AHT (Figs. 1d,f). Does the diffusive perspective provide reasonable representations of AHT changes as well?

We first calculate anomalous northward AHT from anomalous near-surface air temperature according to

$$
F^{\prime}(x)=-\frac{2 \pi p_{s}}{g} c_{p} D_{d}\left(1-x^{2}\right) \frac{d T^{\prime}}{d x} .
$$

The CMIP5 models simulate polar-amplified warming in the Northern Hemisphere and damped warming over the Southern Ocean (Fig. 4a). Given $T^{\prime}(x)$ from CMIP5 models and the value of $D_{d}$ derived from the ERAInterim climatology above, near-surface air temperature diffusion [Eq. (10)] predicts decreased poleward AHT in the Northern Hemisphere midlatitudes (Fig. 3e), at odds with the increased poleward AHT simulated by the models (Figs. 3a,c,d). In the Southern Hemisphere midlatitudes, it predicts increased poleward AHT, consistent with the sign of CMIP5 changes but with insufficient magnitude. Overall, temperature diffusion provides a poor representation of meridional AHT changes.

We next calculate anomalous northward AHT from anomalous near-surface MSE according to

$$
F^{\prime}(x)=-\frac{2 \pi p_{s}}{g} D_{m}\left(1-x^{2}\right) \frac{d h^{\prime}}{d x},
$$

where $h^{\prime}(x)=c_{p} T^{\prime}(x)+L_{v} q^{\prime}(x)$ and $q^{\prime}(x)$ denotes anomalous specific humidity. Assuming constant relative humidity as above, $h^{\prime}(x)$ depends only on $T^{\prime}(x)$ according to the Clausius-Clapeyron relation. ${ }^{3}$ The pattern of $h^{\prime}(x)$ simulated by the CMIP5 models is strikingly different from that of $T^{\prime}(x)$ (Fig. 4a). In the tropics, $h^{\prime}(x) / c_{p}$ is about a factor of 4 greater than $T^{\prime}(x)$, owing to the fact that, from Clausius-Clapeyron, $q(x)$ increases strongly (per degree of warming) where climatological temperatures are warm (Roe et al. 2015). Thus, despite relatively uniform $T^{\prime}(x)$ throughout the tropics and midlatitudes, $h^{\prime}(x)$ is strongly peaked near the equator, enhancing the MSE gradient relative to climatology. At the poles, where temperatures are cold, $h^{\prime}(x) / c_{p}$ is only slightly greater than $T^{\prime}(x)$. Yet, polar warming is sufficiently amplified that the MSE gradient is reduced relative to climatology.

Given $h^{\prime}(x)$ from CMIP5 models and the value of $D_{m}$ derived from the ERA-Interim climatology above, MSE diffusion [Eq. (11)] predicts increased poleward AHT in the midlatitudes of both hemispheres and decreased poleward AHT into the Arctic (thick line in Fig. 3f), qualitatively consistent with CMIP5 changes (Figs. 3a,c,d). Partitioning $F^{\prime}(x)$ into moist and dry components (by use of the Hadley cell parameterization of appendix B) predicts increased poleward latent energy transport compensated by decreased poleward drystatic energy transport in midlatitudes, and increased equatorward latent energy transport compensated by increased poleward dry-static energy transport in the tropics (Fig. 3f)—broadly consistent with CMIP5 changes (Fig. 3d).

From the perspective of MSE diffusion, the meridional structure of anomalous AHT is directly linked to the meridional pattern of $h^{\prime}(x)$. Increased poleward AHT in midlatitudes reflects an increased MSE gradient driven by the larger increase in moisture in the tropics, where climatological temperatures are warm. This is consistent with increased poleward latent energy transport in midlatitudes. Decreased poleward AHT into the Arctic is a consequence of a decreased MSE gradient at high latitudes caused by polar-amplified warming. This is consistent with decreased poleward dry-static energy transport into polar regions. The ability to qualitatively reproduce CMIP5 AHT changes suggests that MSE diffusion provides a decent approximation of meridional AHT. However, the diffusive perspective, as applied here, is also inherently diagnostic given that the pattern

\footnotetext{
${ }^{3}$ The climatological temperature at each latitude is set to the annual-mean ERA-Interim value. Results are similar if the CMIP5 preindustrial climatology is used instead.
} 
of $h^{\prime}(x)$ itself depends on poleward AHT. Moreover, the magnitude of predicted poleward AHT changes are generally too large.

\section{IN SUM}

- The three perspectives-dynamic, energetic, and diffusive - provide complementary descriptions of meridional AHT changes, as demonstrated by their application to abrupt $\mathrm{CO}_{2}$ quadrupling simulations of CMIP5 GCMs. Our use of a consistent set of model simulations permits a novel, like-with-like comparison of the perspectives.

- The decomposition of AHT changes into the individual dynamical contributions agrees with the singlemodel results of $\mathrm{Wu}$ et al. (2011): each contribution varies substantially with latitude but together they sum to produce a net AHT anomaly that is relatively seamless and hemispherically symmetric. Moreover, the individual dynamical contributions to AHT changes do not obviously track changes in atmospheric circulations. We argue that while the dynamic perspective provides a useful diagnostic of AHT changes, it does not offer prognostic understanding of those changes.

- Following Zelinka and Hartmann (2012) and Huang and Zhang (2014), we decompose AHT changes into energetic contributions and find consistent results: AHT primarily mirrors the pattern of $G^{\prime}(x)$. We argue that this result arises from the fact that $R_{f}(x)$ and $\lambda(x) T^{\prime}(x)$ have little meridional structure; this lack of meridional structure in $\lambda(x) T^{\prime}(x)$ arises from a compensation of large $T^{\prime}(x)$ at high latitudes by small $\lambda(x)$ associated with positive feedbacks in polar regions. The energetic perspective naturally explains 1) the hemispherically symmetric increase in midlatitude AHT as a result of hemispherically symmetric $G^{\prime}(x)$, and 2) the decreased AHT into the Arctic as necessary to balance energy input from $R_{f}(x)$ and $G^{\prime}(x)$ with a weak radiative response.

- Inspired by previous work (e.g., North 1981; Flannery 1984; Hwang and Frierson 2010; Roe et al. 2015; Merlis and Henry 2018), we apply the principles of temperature and MSE diffusion directly to CMIP5 patterns of $T^{\prime}(x)$ and $h^{\prime}(x)$, respectively. MSE diffusion qualitatively reproduces AHT changes, while temperature diffusion fails.

The results so far suggest that the dynamic perspective provides only limited understanding of meridional AHT changes, while energetic and diffusive perspectives each provide diagnostic insights into AHT changes in terms of physical processes. In the following section we show that the energetic and diffusive perspectives can be combined to yield a prognostic energy balance model (EBM) that satisfies energetic constraints on the atmospheric column via downgradient energy transport of anomalous MSE. We demonstrate that the EBM with a meridionally uniform value of diffusivity successfully predicts the meridional structure of both AHT and surface temperature changes as simulated by CMIP5 models under $\mathrm{CO}_{2}$ forcing. We will argue that this prognostic success can be linked to a combination of energetic and diffusive constraints and will consider the EBM response to several idealized scenarios that allow us to probe the limits of energetic and diffusive perspectives on AHT changes.

\section{Combining energetic and diffusive perspectives on meridional heat transport changes}

In light of the success of diffusive, downgradient MSE transport as an approximation for climatological and anomalous poleward AHT (Figs. 1 and 3; Hwang and Frierson 2010; Hwang et al. 2011), we combine Eqs. (7), (9), and (11) to produce a steady-state "Moist" EBM that balances anomalous atmospheric heating via diffusion of anomalous MSE:

$$
\lambda(x) T^{\prime}(x)+R_{f}(x)+G^{\prime}(x)=-\frac{p_{s}}{g a^{2}} D_{m} \frac{d}{d x}\left[\left(1-x^{2}\right) \frac{d h^{\prime}}{d x}\right]
$$

The Moist EBM is the same as that used in Roe et al. (2015), Siler et al. (2018), and Bonan et al. (2018).

Given values of $\lambda(x), R_{f}(x)$, and $G^{\prime}(x)$ for each CMIP5 model at a century after abrupt $\mathrm{CO}_{2}$ quadrupling (Fig. 5) and a value of $D_{m}$ derived from the ERA-Interim climatology (section 2c), the Moist EBM simultaneously predicts patterns of $T^{\prime}(x)$ and $F^{\prime}(x)$. We average across ensemble members to produce an EBM-mean response for comparison to the CMIP5-mean response. We further employ the Hadley cell parameterization to partition $F^{\prime}(x)$ into latent and dry-static energy components within the tropics, as above.

We note that $\lambda(x)$ and $G^{\prime}(x)$ contain substantial information about the structure of each GCM's climate response. However, any error in the structure of $T^{\prime}(x)$ implies errors in $\lambda(x) T^{\prime}(x)$ and therefore in AHT changes as well. This formulation of the Moist EBM is thus not as constrained as earlier versions that prescribed radiative fluxes $\left[\lambda(x) T^{\prime}(x)\right]$ associated with each feedback rather than $\lambda(x)$ (Hwang and Frierson 2010; Hwang et al. 2011). Despite this additional degree of freedom, the Moist EBM broadly reproduces the zonalmean climate response as simulated by CMIP5 models (cf. Figs. 4a,b and Figs. 6a-c and 3b-d). In particular, it 

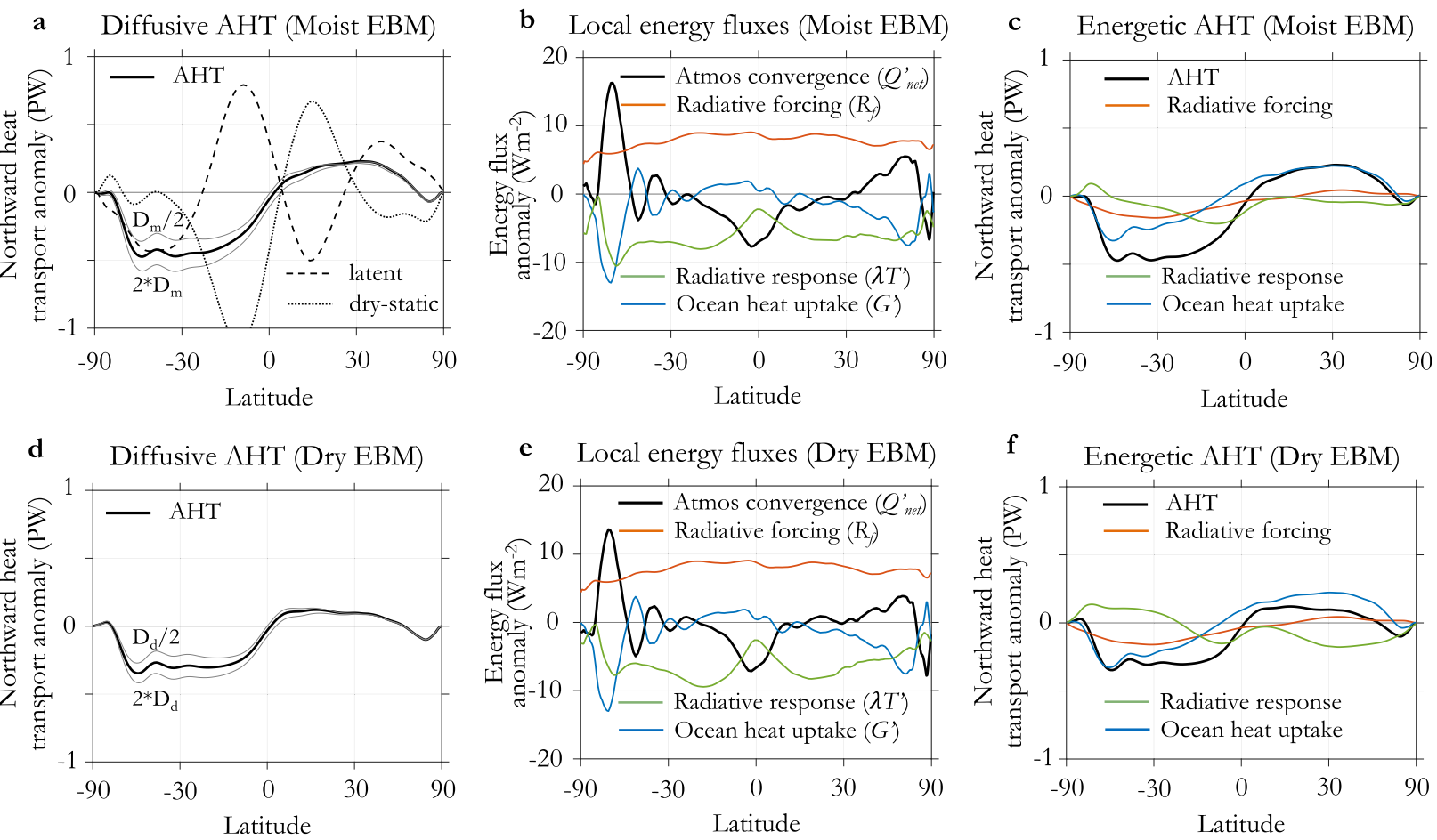

FIG. 6. Combined energetic and diffusive perspectives on anomalous meridional atmospheric heat transport (Moist and Dry EBM responses at year 100 following abrupt $\mathrm{CO}_{2}$ quadrupling). (a) Moist EBM anomalous AHT; thick black, dashed and dotted lines show result with diffusivity derived from the ERA-Interim climatology while thin black line shows results with diffusivity half that value. (b) Moist EBM anomalous zonal-mean energy fluxes into the atmospheric column from radiative forcing, radiative response, surface heat fluxes (ocean heat uptake), and atmospheric heat flux convergence. (c) Moist EBM anomalous AHT derived by meridionally integrating anomalous zonal-mean energy fluxes according to Eq. (8); red line shows anomalous AHT implied by radiative forcing; blue line shows anomalous AHT implied by ocean heat uptake; green line shows anomalous AHT implied by radiative response (feedbacks); black line shows net AHT implied as the sum of the others. (d)-(f) As in (a)-(c), but for Dry EBM.

predicts seamless meridional AHT anomalies, with increased poleward AHT in the midlatitudes of both hemispheres (by about $0.3 \mathrm{PW}$ at the climatological maxima) and slightly decreased poleward AHT into the Arctic (Fig. 6a). Moreover, it predicts polar amplified warming in the Arctic and damped warming over the Southern Ocean (Fig. 4b). Consistent with previous studies (Rose et al. 2014; Roe et al. 2015; Siler et al. 2018; Bonan et al. 2018), the Moist EBM accurately captures the climate response as simulated by individual GCMs as well (Fig. $\mathrm{C} 1$ in appendix C).

The Moist EBM also reproduces the CMIP5 partitioning between latent and dry-static energy transport (cf. Figs. 6a and 3d). Increased poleward latent energy transport is compensated by decreased poleward drystatic energy transport in midlatitudes, while increased equatorward latent energy transport is compensated by increased poleward dry-static energy transport in the tropics where the Hadley cell parameterization is active.

Like the CMIP5 response, the meridional structure of $F^{\prime}(x)$ predicted by the Moist EBM primarily reflects that required by $G^{\prime}(x)$ (Figs. 6b,c); the radiative response $\lambda(x) T^{\prime}(x)$ varies little with latitude, implying little impact on $F^{\prime}(x)$, while variations in $R_{f}(x)$ with latitude imply a slight increase in poleward AHT in midlatitudes (Figs. 6b,c). On their own, energetic considerations do not provide insight into the pattern of warming. However, given the additional knowledge that meridional AHT changes are accomplished by diffusive, downgradient MSE transport, the structure of $F^{\prime}(x)$ can be viewed as implying a specific pattern of $h^{\prime}(x)$ and thus $T^{\prime}(x)$ (Fig. 4b).

Alternatively, the meridional structure of $F^{\prime}(x)$ can be viewed as a consequence of anomalous MSE gradients. Consider an initial meridionally uniform perturbation in temperature. It will be associated with large $h^{\prime}(x)$ in the tropics but small $h^{\prime}(x)$ in polar regions due to a preferential increase in $q^{\prime}(x)$ at warmer temperatures due to the Clausius-Clapeyron relation (Fig. 7a). Perfectly efficient downgradient transport of MSE would completely flatten the anomalous MSE gradient, and would necessarily result in polar amplification (Fig. 7b; Merlis and Henry 2018). For a system of finite diffusivity the ultimate balance will tend toward somewhere between 

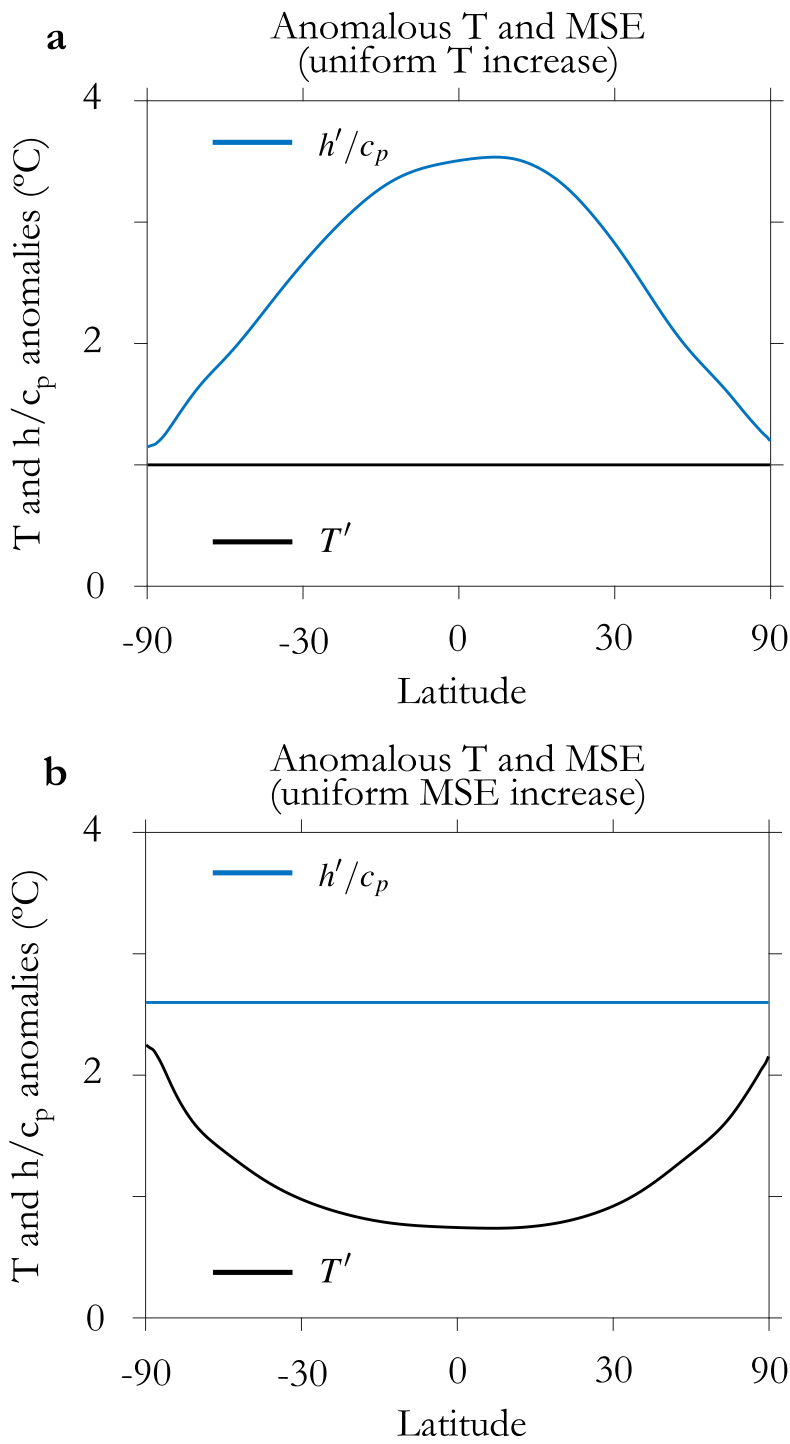

FIG. 7. Idealized anomalies associated with a meridionally uniform increase in near-surface air temperature and a meridionally uniform increase in moist static energy. (a) Uniform near-surface air temperature increase of $1^{\circ} \mathrm{C}$ (black line) and associated MSE increase (divided by $c_{p}$; blue line). (b) Uniform MSE increase (divided by $c_{p}$; blue line) by the same global-mean value as in (a) and associated near-surface air temperature increase (black line). Hemispheric asymmetries reflect climatological hemispheric asymmetries in near-surface air temperature in ERA-Interim (Fig. 2)

these extremes, with a tropical peak in MSE, increased poleward AHT in midlatitudes, and some intermediate polar amplification of temperature. Indeed, increased poleward AHT in midlatitudes within the Moist EBM reflects an enhanced MSE gradient in the tropics (Fig. 4b). Decreased poleward AHT into the Arctic reflects a reduced MSE gradient associated with polar amplification that exceeds that in Fig. 7b. In turn, these meridional AHT changes shape the pattern of $T^{\prime}(x)$ and thus radiative response $\lambda(x) T^{\prime}(x)$ so that local energy balance is achieved (Fig. 6b).

The above arguments represent two distinct perspectives on what governs temperature and AHT changes. From the energetic perspective, the meridional structure of $F^{\prime}(x)$ is constrained by TOA radiation and surface energy fluxes while $T^{\prime}(x)$ and $h^{\prime}(x)$ must adjust such that those meridional AHT changes are realized. From the diffusive perspective, the relationship between $T^{\prime}(x)$ and $h^{\prime}(x)$ implies meridional AHT changes with warming while TOA radiation responds accordingly such that local energy balance is achieved. A key question is this: Which perspective more accurately describes constraints on the meridional patterns of $T^{\prime}(x)$ and $F^{\prime}(x)$ ? By construction, the Moist EBM satisfies both energetic and diffusive constraints at once, and thus is an ideal test bed for examining their relative roles.

A strong indication comes from comparing meridional AHT changes predicted by the Moist EBM with those inferred by applying the diffusive perspective in section 3c. When diagnosed directly from the CMIP5 pattern of $h^{\prime}(x)$ using Eq. (11) and the value of $D_{m}$ derived from the ERA-Interim climatology, the magnitude of $F^{\prime}(x)$ was too large at most latitudes (cf. Figs. 3c,f). Yet, the Moist EBM using the same value of $D_{m}$ accurately predicts the CMIP5 pattern of $F^{\prime}(x)$ (cf. Fig. 3c and thick line in Fig. 6a). Importantly, this improvement in AHT comes at the expense of introducing errors in predicted $T^{\prime}(x)$ with too little warming in the Arctic most notably (cf. Figs. 4a,b). That is, when allowed to adjust within a self-consistent EBM framework, $F^{\prime}(x)$ becomes aligned with that implied by energetic constraints (Fig. 3c) while $h^{\prime}(x)$ and $T^{\prime}(x)$ adjust away from CMIP5 values in order to realize that meridional pattern of $F^{\prime}(x)$.

This key result can be understood from energetic arguments as well. Where radiative response to surface warming is inefficient $[\lambda(x)$ near zero], such as in polar regions, $R_{f}(x)$ and $G^{\prime}(x)$ must together be balanced primarily by atmospheric heat flux divergence. In turn, the pattern of $T^{\prime}(x)$ must adjust such that the anomalous MSE gradient yields the required $F^{\prime}(x)$ [Eq. (11)]. Thus, $T^{\prime}(x)$ in polar regions is sensitive to the details linking AHT changes to anomalous gradients in MSE, while $F^{\prime}(x)$ itself is not. This picture approximately holds outside of polar regions as well since meridional variations in $\lambda(x) T^{\prime}(x)$ are relatively small compared to those of $G^{\prime}(x)$-reflecting a relatively weak relationship between the meridional pattern of warming and the meridional pattern of radiative response. That is, a variety of $T^{\prime}(x)$ patterns can produce similar patterns of $F^{\prime}(x)$ because TOA radiation is relatively insensitive to $T^{\prime}(x)$. 
In turn, the pattern of $T^{\prime}(x)$ depends sensitively on the relationship between $F^{\prime}(x)$ and $T^{\prime}(x)$.

It thus appears that meridional AHT is governed by energetic constraints while $T^{\prime}(x)$ must adjust according to the details of how it is related to $F^{\prime}(x)$. The above interpretation is expected to hold so long as the atmosphere behaves sufficiently diffusively; in the limit of small $D_{m}$, meridional variations in $\lambda(x) T^{\prime}(x)$ become large relative to changes in atmospheric heat flux divergence and $F^{\prime}(x)$ becomes sensitive to both the value of $D_{m}$ and the pattern of $\lambda(x)$.

In what follows, we consider four idealized scenarios that probe the limits of the above interpretation:

1) The Moist EBM response using a value of diffusivity increased or decreased by a factor of 2 . This explores the sensitivity of the climate response to a different diffusive representation of meridional AHT under the same energetic constraints as above. How would the meridional patterns of $T^{\prime}(x)$ and $F^{\prime}(x)$ be different in these scenarios?

2) The EBM response to $\mathrm{CO}_{2}$ forcing in the limit of zero relative humidity, representing diffusive, downgradient transport of dry-static energy. This "Dry" EBM explores the sensitivity of the climate response to a vastly different representation of meridional AHT under the same energetic constraints as above. What would the energetic and diffusive perspectives predict for the meridional patterns of $T^{\prime}(x)$ and $F^{\prime}(x)$ in this scenario?

3) The Moist and Dry EBM response to $\mathrm{CO}_{2}$ forcing when $G^{\prime}(x) \approx 0$, representing a near-equilibrium response. This explores the climate response when ocean heat uptake (the primary energetic constraint on meridional AHT changes in transient CMIP5 simulations) no longer plays a role. How would this modify the meridional patterns of $T^{\prime}(x)$ and $F^{\prime}(x)$ ?

4) The Moist and Dry EBM response to spatially uniform forcing and feedbacks. This explores the climate response when all meridional structure in energetic constraints on AHT changes is eliminated. What governs $T^{\prime}(x)$ and $F^{\prime}(x)$ in this limit?

\section{a. Climate response with diffusivity increased or decreased by a factor of 2}

The Moist EBM [Eq. (12)] accurately predicts the CMIP5 pattern of $F^{\prime}(x)$ when using the value of $D_{m}$ derived from the ERA-Interim climatology (cf. Fig. 3c and thick line in Fig. 6a). Increasing or decreasing $D_{m}$ by a factor of 2 leads to changes in the the structure of $F^{\prime}(x)$ that are much smaller than a factor of 2 (thin lines in Fig. 6a). This is particularly true in the Northern Hemisphere where $\lambda(x)$ is closer to zero and so that changes in the radiative response are greatly muted. The relatively small changes in $F^{\prime}(x)$ arise because the change in $D_{m}$ is compensated by changes in the meridional gradients of $T^{\prime}(x)$ and $h^{\prime}(x)$ (thin lines in Fig. 4b). This supports the finding that poleward AHT changes must satisfy net energetic constraints and are largely insensitive to the details of the diffusive approximation. The ability of the Moist EBM to produce realistic patterns of $T^{\prime}(x)$ and $F^{\prime}(x)$ simultaneously over a wide range of $D_{m}$ values indicates that diffusive, downgradient transport of MSE is a decent approximation of meridional AHT in comprehensive GCMs.

\section{b. Climate response of a Dry EBM}

We combine Eqs. (7), (9), and (10) to produce a Dry EBM that balances anomalous atmospheric heating via anomalous diffusion of dry-static energy:

$$
\lambda(x) T^{\prime}(x)+R_{f}(x)+G^{\prime}(x)=-\frac{p_{s} c_{p}}{g a^{2}} D_{d} \frac{d}{d x}\left[\left(1-x^{2}\right) \frac{d T^{\prime}}{d x}\right] .
$$

The Dry EBM is the same as traditionally used in EBM studies (e.g., Budyko 1969; Sellers 1969; Stone 1978; North 1975, 1981).

Given values of $R_{f}(x), \lambda(x)$, and $G^{\prime}(x)$ for each CMIP5 model at a century after abrupt $\mathrm{CO}_{2}$ quadrupling (Fig. 5) and the value of $D_{d}$ derived from the ERA-Interim climatology, the Dry EBM simultaneously predicts patterns of $T^{\prime}(x)$ and $F^{\prime}(x)$. As above, we average across ensemble members to produce an EBM-mean response.

It is difficult to anticipate the Dry EBM response from diffusive arguments alone-AHT changes must reflect the meridional pattern of $T^{\prime}(x)$, but will warming be tropically or polar amplified? However, based on the constraints of energy input into the tropical and polar atmosphere by $R_{f}(x)$ and $G^{\prime}(x)$ combined with a relatively weak radiative response to warming, energetic reasoning anticipates increased poleward AHT in midlatitudes and decreased poleward AHT into the Arctic; in turn, increased poleward AHT in midlatitudes would imply tropically amplified $T^{\prime}(x)$. A lack of polaramplified warming would result in only slightly reduced radiative response at high latitudes [due to small $\lambda(x)$ ], demanding only a slightly smaller increase in poleward AHT to maintain local energy balance relative to CMIP5 models.

Indeed, the Dry EBM produces increased poleward AHT in the midlatitudes of both hemispheres, and slightly decreased poleward AHT into the Arctic (Fig. 6d), similar to the patterns of $F^{\prime}(x)$ in CMIP5 models (Figs. 3a,c) and the Moist EBM (Fig. 6a). Note that predicted $F^{\prime}(x)$ is much improved compared to that 
derived by applying temperature diffusion directly to the CMIP5 patterns of $T^{\prime}(x)$ (Fig. 3e). Importantly, this improvement that comes at the expense of the Dry EBM failing to reproduce the polar-amplified pattern of warming in CMIP5 models (cf. Figs. 4a,c). As above, this result is insensitive to the value of diffusivity used: increasing or decreasing $D_{d}$ by a factor of 2 does not substantially change the structure of $F^{\prime}(x)$ (thin lines in Fig. 6d) or $T^{\prime}(x)$ (thin lines in Fig. 4c).

These results suggest that the energetic perspective offers prognostic insights: poleward AHT changes must satisfy the net energetic demands of radiative forcing and ocean heat uptake, and are only weakly influenced by the radiative response to the meridional pattern of warming. In turn, the meridional pattern of surface warming must adjust to produce meridional AHT changes that satisfy these energetic constraints. Without changes in latent energy transport, the climate response to greenhouse gas forcing would be tropically amplified in order to accomplish the required meridional AHT changes.

\section{c. Climate response at near-equilibrium}

We next consider the climate response to greenhouse gas forcing when $G^{\prime}(x) \approx 0$, representing nearequilibrium conditions. We compare the response of the Moist and Dry EBMs [Eqs. (12) and (13), respectively] to the equilibrium response of a mixed layer (slab) ocean version of the Community Atmosphere Model version 4 (CAM4; Neale et al. 2013) driven by a doubling of $\mathrm{CO}_{2}$ above preindustrial levels. CAM4's patterns of $R_{f}(x), \lambda(x)$, and $G^{\prime}(x)$ are shown in Fig. 5 (dashed lines). The pattern of $R_{f}(x)$ is similar to that of the CMIP5 mean (though half the magnitude due to $\mathrm{CO}_{2}$ doubling rather than quadrupling). The pattern of $\lambda(x)$ is qualitatively similar to that of the CMIP5 mean, but shows more negative values in the tropics and more positive values in the Southern Hemisphere high latitudes. ${ }^{4}$ The value of $G^{\prime}(x)$ is exactly zero throughout the tropics and midlatitudes, but it has nonzero values near the poles due to a change in surface heat fluxes arising from a decrease in the growth, equatorward transport, and melt of sea ice.

What changes in meridional AHT can be anticipated from energetic constraints? The meridional pattern of

\footnotetext{
${ }^{4}$ More positive high-latitude feedbacks in CAM4 are likely the result of enhanced polar surface warming relative to CMIP5 models (cf. Figs. 8a and 4a): as high-latitude surface warming increases, positive sea ice albedo feedbacks become activated and atmospheric warming becomes more confined to the lower troposphere leading to a more positive local lapse-rate feedback (Po-Chedley et al. 2018).
}

$R_{f}(x)$ implies a slight increase in poleward AHT in midlatitudes, similar to the CMIP5 models (Huang and Zhang 2014; see Fig. 3c herein). However, the main driver of increased midlatitude poleward AHT in the transient CMIP5 simulations-subpolar ocean heat uptake (Huang and Zhang 2014; see Fig. 3c herein)-is absent in the equilibrium CAM4 simulation. This suggests that $F^{\prime}(x)$ may instead track more closely with that implied by the meridional pattern of the radiative response $\lambda(x) T^{\prime}(x)$. In turn, much more negative values of $\lambda(x)$ in the tropics than at high latitudes in CAM4 suggest a much larger radiative response to warming in the tropics than at high latitudes. Thus, from energetic considerations we can qualitatively expect a smaller increase or, perhaps, a decrease of midlatitude poleward AHT. Meanwhile, $G^{\prime}(x)$ nearly balances $R_{f}(x)$ in the Arctic within CAM4 (Fig. 5a), suggesting that the radiative response to Arctic warming, however weak, must be balanced by increased poleward AHT

These anticipated changes are broadly confirmed by CAM4's response to $\mathrm{CO}_{2}$ forcing (Fig. 8). While warming is strongly polar amplified in both hemispheres (Fig. 8a), poleward AHT decreases in the midlatitudes and increases into polar regions (Fig. 8b) -opposite in sign to the poleward AHT changes seen under transient warming of CMIP5 models (Fig. 3) but broadly consistent with energetic expectations. Where $G^{\prime}(x) \approx 0$ (tropics and midlatitudes; Figs. 5a and 8b), the pattern of anomalous atmospheric energy divergence must exactly mirror net TOA radiation changes $\left[R_{f}(x)+\lambda(x) T^{\prime}(x)\right]$. Because the pattern of $R_{f}(x)$ varies relatively little with latitude compared to the pattern of $\lambda(x) T^{\prime}(x)$ (Fig. 8b), energy is anomalously transported from regions of positive feedbacks to regions of negative feedbacks, consistent with the findings of Feldl and Roe (2013a). ${ }^{5}$ Indeed, while meridional variations in $R_{f}(x)$ imply a slight increase in poleward AHT in midlatitudes, the pattern of $F^{\prime}(x)$ largely tracks that implied by the larger meridional variations in $\lambda(x) T^{\prime}(x)$, resulting in decreased poleward AHT in midlatitudes. Nonzero values of $G^{\prime}(x)$ near the poles (Figs. 5a and 8b) result in increased poleward AHT in polar regions (Fig. 8c).

Given CAM4's values of $R_{f}(x), \lambda(x)$, and $G^{\prime}(x)$ (Fig. 5), the Moist EBM accurately captures CAM4's response, with decreased poleward AHT in midlatitudes and increased poleward AHT into polar regions (Fig. 8f). Moreover, it broadly reproduces CAM4's meridional patterns of $T^{\prime}(x)$ and $h^{\prime}(x)$ (Fig. 8d), although

\footnotetext{
${ }^{5}$ Roe et al. (2015) and Feldl et al. (2017b) further showed that $F^{\prime}(x)$ adjusts accordingly as individual radiative feedbacks (e.g., sea ice albedo) are modified within atmospheric GCMs.
} 

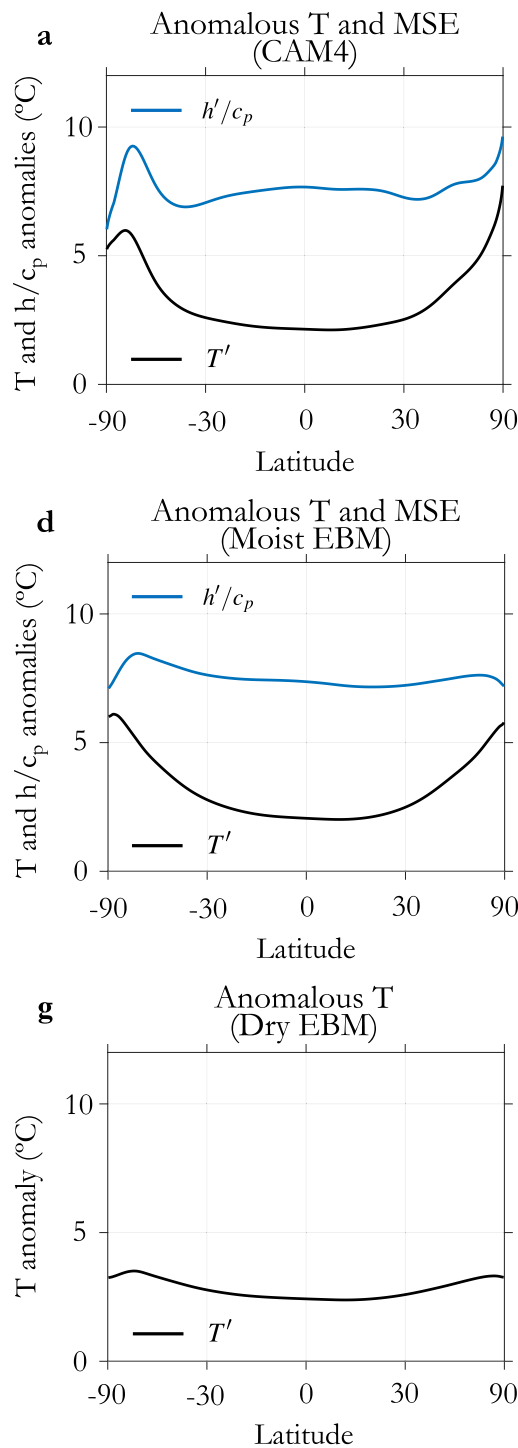

b Local energy fluxes (CAM4)

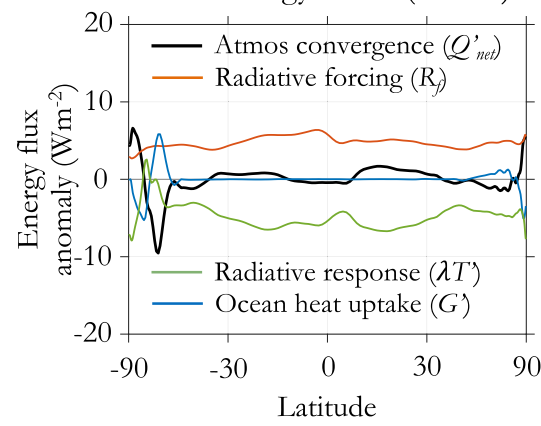

e Local energy fluxes (Moist EBM)

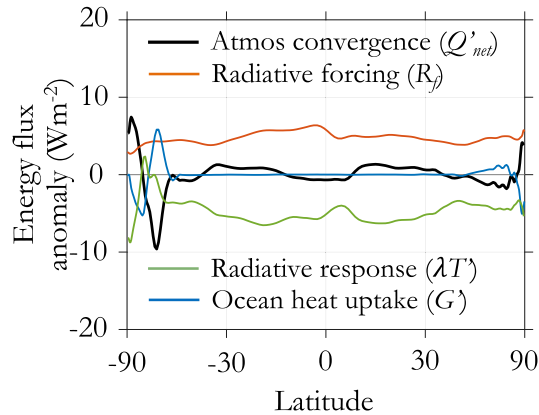

h Local energy fluxes (Dry EBM)

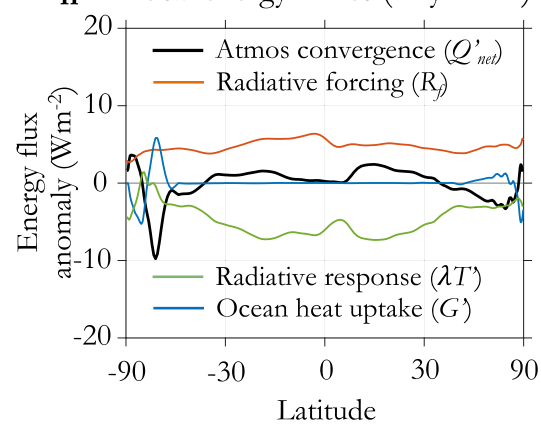

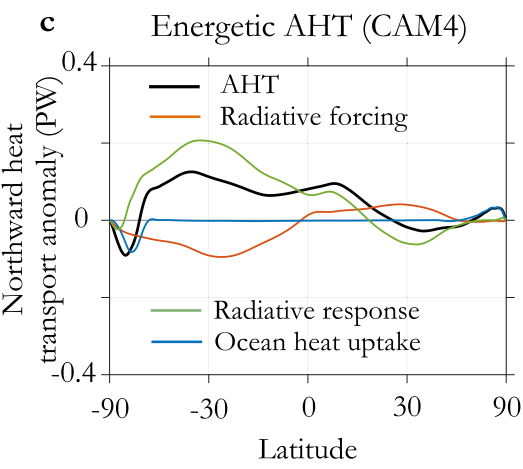

f Energetic AHT (Moist EBM)

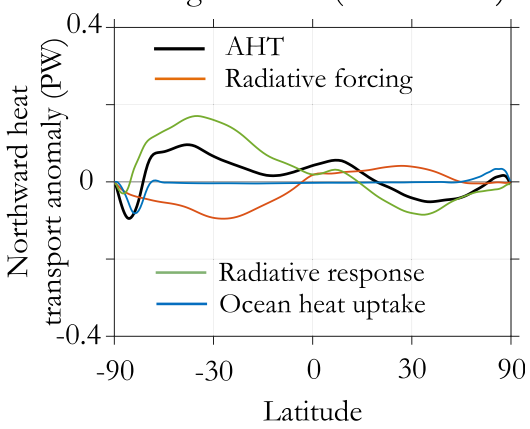

i Energetic AHT (Dry EBM)

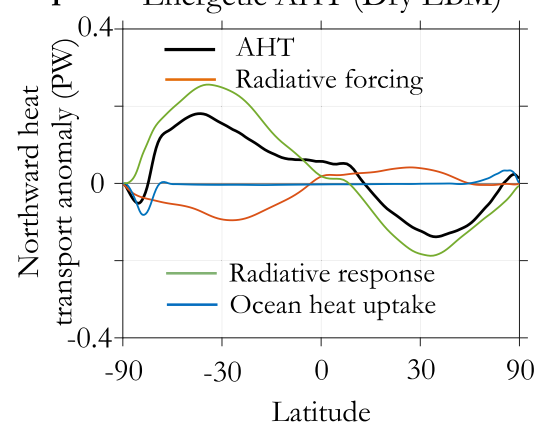

FIG. 8. Near-equilibrium climate response to $\mathrm{CO}_{2}$ doubling (CAM4, Moist EBM, and Dry EBM). (a) CAM4 anomalous near-surface air temperature (black line) and MSE (divided by $c_{p}$; blue line). (b) CAM4 anomalous zonal-mean energy fluxes into the atmospheric column from radiative forcing, radiative response, surface heat fluxes (ocean heat uptake), and atmospheric heat flux convergence. (c) CAM4 anomalous AHT derived by meridionally integrating anomalous zonal-mean energy fluxes according to Eq. (8); red line shows anomalous AHT implied by radiative forcing; blue line shows anomalous AHT implied by ocean heat uptake; green line shows anomalous AHT implied by radiative response (feedbacks); black line shows net AHT implied as the sum of the others. (d)-(f) As in (a)-(c), but for Moist EBM. (g)-(i) As in (a)-(c), but for Dry EBM.

predicted Arctic warming is too small. The mismatch with CAM4's pattern of $h^{\prime}(x)$ in the Arctic, while still producing similar patterns of $F^{\prime}(x)$, suggests that the diffusive approximation for AHT is inadequate at these latitudes; the source of this discrepancy warrants further study.

Like the Moist EBM, the Dry EBM qualitatively captures CAM4's pattern of $F^{\prime}(x)$, with decreased poleward AHT in midlatitudes and increased poleward AHT into polar regions (Fig. 8i), as expected from energetic constraints. However, it is unable to reproduce CAM4's meridional pattern of $T^{\prime}(x)$ (Fig. 8g), showing far too little warming at both poles.

The difference between Moist and Dry EBM responses can be readily understood from the energetic perspective. Absent ocean heat uptake, the meridional pattern of $F^{\prime}(x)$ mirrors that implied by $\lambda(x) T^{\prime}(x)$ (Figs. 8f,i). In turn, the meridional pattern of $\lambda(x) T^{\prime}(x)$ primarily mirrors that of $\lambda(x)$, which is the same in both Moist and Dry EBMs. This follows from the fact that 

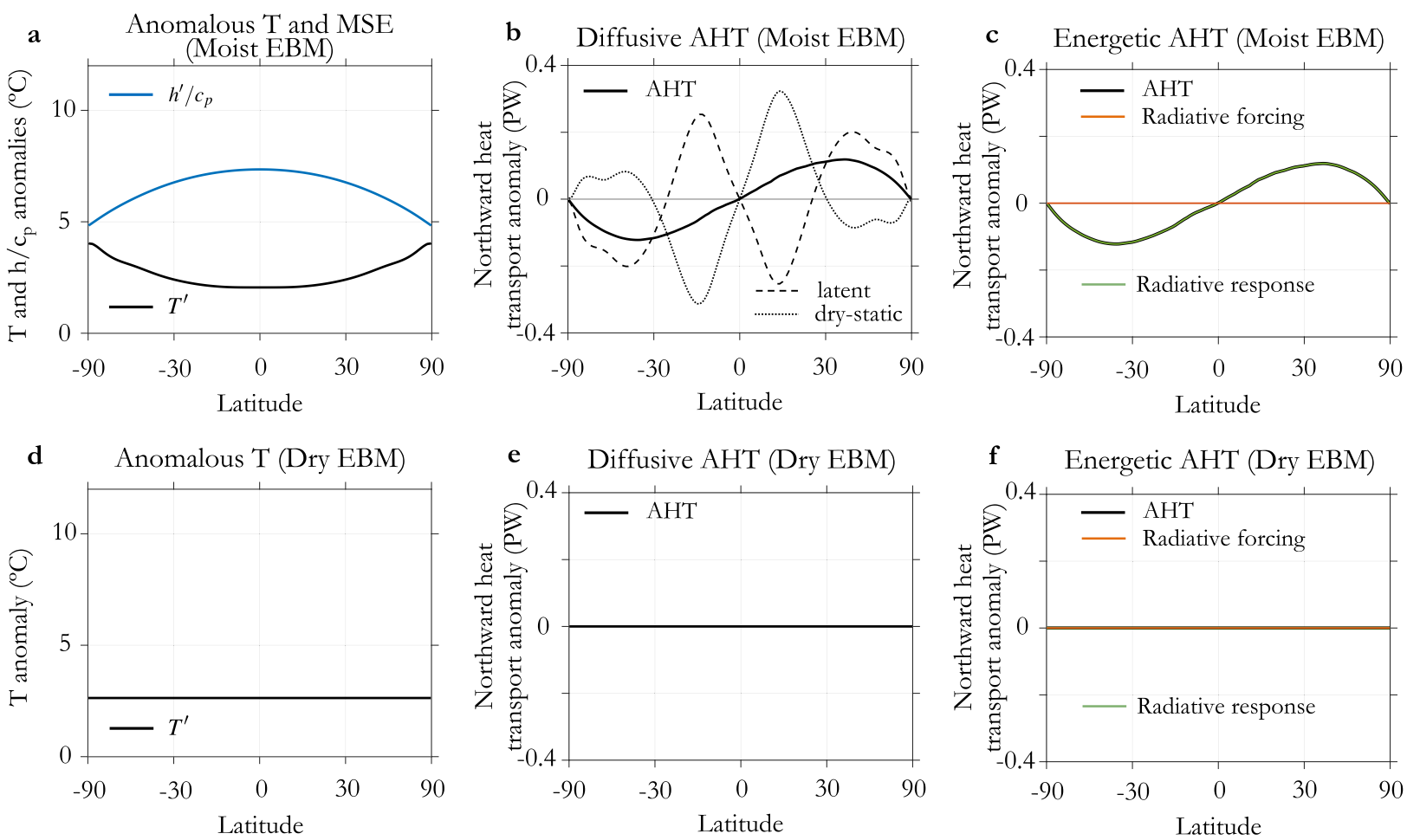

FIG. 9. Climate response to uniform forcing with uniform feedback (Moist EBM and Dry EBM). (a) Anomalous near-surface air temperature (black line) and MSE (divided by $c_{p}$; blue line). (b) Anomalous zonal-mean energy fluxes into the atmospheric column from radiative forcing, radiative response, and atmospheric heat flux convergence. (c) Anomalous AHT derived by meridionally integrating anomalous zonal-mean energy fluxes according to Eq. (8); red line shows anomalous AHT implied by radiative forcing; green line shows anomalous AHT implied by radiative response (feedbacks); black line shows net AHT implied as the sum of the others. (d)-(f) As in (a)-(c), but for Dry EBM.

$T^{\prime}(x)$ varies fractionally much less with latitude compared to $\lambda(x)$. Relatively uniform $T^{\prime}(x)$ within the tropics and midlatitudes thus results in similar meridional patterns of radiative response $\lambda(x) T^{\prime}(x)$ within Moist and Dry EBMs, while large differences in $T^{\prime}(x)$ between Moist and Dry EBMs in polar regions result in muted differences in $\lambda(x) T^{\prime}(x)$ because $\lambda(x)$ is small at high latitudes (Figs. 8e,h). The result is qualitatively similar patterns of $F^{\prime}(x)$ between Moist and Dry EBMs (Figs. 8f,i). By accounting for latent energy transport, the Moist EBM produces $F^{\prime}(x)$ via a strongly polaramplified pattern of $T^{\prime}(x)$ [a weakly polar-amplified pattern of $h^{\prime}(x)$ ]. By disregarding latent energy transport, the Dry EBM accomplishes $F^{\prime}(x)$ via a weakly polar-amplified pattern of $T^{\prime}(x)$.

\section{d. Climate response under uniform forcing and feedbacks}

Finally, we consider the climate responses of the Moist and Dry EBMs under meridionally uniform radiative forcing and feedbacks. We use global-mean values of $R_{f}(x)$ and $\lambda(x)$ taken from CAM4 (Fig. 5) while setting $G^{\prime}(x)=0$. In this case, there are no a priori energetic constraints on the meridional pattern of $F^{\prime}(x)$. The solution of the Dry EBM can be anticipated from either energetic or diffusive perspectives: uniform warming with no change in meridional AHT (Figs. 9d-f). However, anticipating the solution of the Moist EBM requires knowing the details of meridional AHT relates to temperature: a preferential increase in tropical $q^{\prime}(x)$ with warming (Fig. 7a) combined with diffusive, downgradient MSE transport can be expected to produce increased poleward AHT; in turn, a polar-amplified warming pattern is needed to regain local energy balance via radiative response. Indeed, the Moist EBM produces polar-amplified warming with increased poleward AHT at all latitudes (Figs. 9a-c) ${ }^{6}$

In the limit of weak meridional structure in forcing and feedbacks, Dry and Moist EBMs produce distinct patterns of both $T^{\prime}(x)$ and $F^{\prime}(x)$, suggesting that $T^{\prime}(x)$ and $F^{\prime}(x)$ depend sensitively on the details of how meridional AHT is related to temperature. This stands in

\footnotetext{
${ }^{6}$ See Merlis and Henry (2018) for analytic solutions to the Moist EBM under uniform forcing and feedbacks.
} 
stark contrast to the response when there is strong meridional structure in forcing, feedbacks, or ocean heat uptake, as in the CMIP5 models and CAM4. Then, Dry and Moist EBMs produce distinct patterns of $T^{\prime}(x)$ but similar patterns of $F^{\prime}(x)$, suggesting that $T^{\prime}(x)$ depends sensitively on the details of meridional AHT while $F^{\prime}(x)$ appears instead to be energetically constrained.

Comparing Figs. 6, 8, and 9 further suggests that while the magnitude of polar amplification of surface warming depends on the meridional pattern of $\lambda(x)$, there is a strong tendency for polar amplification regardless of the structure of $\lambda(x)$. When $\lambda(x)$ is more positive at high latitudes than elsewhere, strong polar amplification occurs with decreased (or little change in) poleward AHT into polar regions under transient warming (Figs. 6a-c) or with increased poleward AHT into polar regions at near-equilibrium (Figs. 8a-f). When $\lambda(x)$ is spatially uniform, polar amplification occurs with increased poleward AHT into polar regions (Figs. 9a-c). While comprehensive GCMs (Fig. 5b) and observations (Zhang et al. 2018) suggest that substantially more negative values of $\lambda(x)$ at the poles than in the tropics are unrealistic, aquaplanet GCMs without a sea ice albedo feedback that produce such feedback patterns often simulate little to no polar amplification (e.g., Feldl et al. 2017b). Additional Moist EBM simulations (not shown) employing such a $\lambda(x)$ pattern likewise produce meridionally uniform warming as midlatitude poleward AHT increases strongly to balance the tropical energy input and large build-up of tropical MSE. That is, MSE diffusion strongly favors polar amplification and acts to curb tropical amplification. On the other hand, the Dry EBM results suggest that without moisture, the response would favor only weakly polar-amplified or perhaps even tropically amplified warming, depending on the feedback pattern. These results suggest that polar amplification is a nearly inevitable feature of a moist, diffusive atmosphere's response to $\mathrm{CO}_{2}$ forcing (for realistic patterns of radiative feedbacks).

\section{IN SUM}

- The Moist EBM accurately reproduces AHT and $T^{\prime}(x)$ as seen in CMIP5 GCMs under abrupt $\mathrm{CO}_{2}$ quadrupling. AHT changes primarily mirror the pattern of $G^{\prime}(x)$.

- While MSE diffusion applied directly to GCM values of $h^{\prime}(x)$ produced errors in AHT changes (Fig. 3f), the Moist EBM accurately reproduces GCM AHT changes at the expense of introducing small errors in $h^{\prime}(x)$ and $T^{\prime}(x)$ (Figs. 4b and 6a-c). Likewise, while temperature diffusion applied directly to GCM values of $T^{\prime}(x)$ failed entirely to reproduce AHT changes (Fig. 3e), the Dry EBM accurately reproduces GCM
AHT changes at the expense of introducing major errors in $T^{\prime}(x)$ (tropical amplification of warming; Figs. $4 \mathrm{c}$ and $6 \mathrm{~d}-\mathrm{f})$.

- These results suggest that AHT changes are constrained by energetics and relatively insensitive to dynamical details. However, the pattern of $T^{\prime}(x)$ depends sensitively on the details linking it to AHT changes. Given that AHT is well approximated by downgradient MSE transport, we argue that (for realistic feedback patterns) polar amplification is necessary to satisfy energetically constrained AHT changes.

- The Moist EBM is able to reproduce changes seen in equilibrium (slab ocean) simulations with CAM4. With near-zero $G^{\prime}(x)$, AHT changes primarily mirror the pattern of $\lambda(x) T^{\prime}(x)$, in good agreement with previous equilibrium GCM simulations (Feldl and Roe 2013a; Roe et al. 2015; Feldl et al. 2017b).

- Uniform forcing and feedback simulations with the Moist and Dry EBMs (e.g., Merlis and Henry 2018) reveal that without spatial structure in $G^{\prime}(x)$ or $\lambda(x)$, both $T^{\prime}(x)$ and AHT changes depend sensitively on the details of how meridional AHT is related to temperature and moisture.

\section{Discussion and conclusions}

The results presented here suggest that meridional AHT and its changes can be naturally understood from the energetic perspective. Meridional AHT must, on long time scales, act to balance the zonal-mean heating of the atmospheric column by net TOA radiation and surface energy fluxes [Eq. (4)]. In turn, the energetic perspective permits diagnostic quantification of climatological AHT in terms of the transport implied by TOA radiation and surface heat fluxes (section $2 b$; Trenberth and Caron 2001) and of AHT changes in terms of the transport implied by radiative forcing, radiative response, and ocean heat uptake (section 3b; Zelinka and Hartmann 2012; Huang and Zhang 2014). In this view, meridional AHT most closely mirrors energetic contributions that have greatest meridional variation: TOA radiation in the climatology, ocean heat uptake in the transient forced response of coupled (CMIP5) GCMs, and radiative response in the equilibrium forced response of an atmospheric GCM (slab ocean CAM4).

The energetic perspective offers prognostic insights into AHT changes when combined with a simple, diffusive representation of AHT to form a self-consistent EBM [section 4; Eq. (12)]. Under a wide range of diffusivity values (section 4a), and even in the limit that latent energy transport is ignored (section $4 b$ ), the EBM produces meridional AHT changes that well 
approximate those of coupled and atmospheric GCMs under $\mathrm{CO}_{2}$ forcing. The results suggest that meridional AHT changes are strongly constrained by the meridional patterns of forcing, feedbacks and ocean heat uptake and are largely insensitive to the details of how that AHT is accomplished. These findings hold so long as these energetic constraints have substantial meridional structure, as is seen in comprehensive GCMs.

In this view, the ability of the Moist EBM to predict meridional AHT changes simulated by GCMs reflects its realization of energetic constraints [left-hand side of Eq. (12)]. Its ability to simultaneously predict the meridional patterns of warming simulated by GCMs is evidence that diffusion of near-surface MSE is a decent approximation to the relationship between meridional AHT and surface temperature changes [right-hand side of Eq. (12)]. ${ }^{7}$ The success of the diffusive approximation is further evidenced by its decent representation of observed climatological AHT and its partitioning between latent and dry-static energy fluxes (section 2c). Meridional AHT thus appears to be constrained by energetics while being mediated by large-scale diffusion of MSE. These findings align with early studies (e.g., North 1981) that framed AHT in terms of energetic constraints and employed temperature diffusion as simple way to describe AHT. What is added here is the demonstration that this energetic-diffusive perspective captures the response seen in comprehensive GCMs, particularly when the critical role of moisture is accounted for so that the diffusion acts on gradients in MSE rather than temperature.

A traditional description of the role of meridional AHT in shaping the pattern of surface warming is in terms of changes in atmospheric energy flux convergence at a given latitude (e.g., Fig. 3b), permitting a diagnosis of its contribution to zonal-mean warming by dividing by the Planck response (e.g., Crook et al. 2011; Feldl and Roe 2013a; Pithan and Mauritsen 2014; Goosse et al. 2018). In this view, the fact that poleward AHT into the Arctic changes little, or even decreases, under greenhouse gas forcing in CMIP5 models implies that it plays little to no role in Arctic warming. Instead, Arctic amplification has been suggested to be a consequence of a weaker radiative response to surface warming [more positive $\lambda(x)$ ] in polar regions

\footnotetext{
${ }^{7}$ Permitting the diffusivity to change with latitude or with climate state (e.g., Shaw and Voigt 2016) could potentially improve the ability of the EBM to reproduce the CMIP5 results. Note that the Moist EBM could alternatively be constructed as a Dry EBM with a diffusivity that varies meridionally based on the climatological temperature (e.g., Rose et al. 2014; Merlis and Henry 2018).
}

than at lower latitudes (Kay et al. 2012; Pithan and Mauritsen 2014).

The results presented here challenge this description. The Moist EBM predicts amplified Arctic warming, in good agreement with CMIP5 models, when the CMIP5 meridional pattern of $\lambda(x)$ is employed (Figs. 6a-c); diagnosing contributions to zonal-mean warming within the Moist EBM would lead to the same conclusions regarding the role of AHT changes in Arctic amplification as reported for CMIP5 models (Pithan and Mauritsen 2014; Goosse et al. 2018). However, the Moist EBM also predicts amplified Arctic warming for meridionally uniform $\lambda(x)$ (Figs. 9a-c). This suggests that while the degree of polar amplification depends on the meridional pattern of $\lambda(x)$, the presence of polar amplification itself is a nearly inevitable feature of a macroturbulent, moist atmosphere's response to greenhouse gas forcing. Only when latent energy is neglected (as in the Dry EBM), when subpolar ocean heat uptake is large (Southern Ocean response of CMIP5 models), when polar feedbacks are unrealistically negative, or when forcing is localized in the tropics (Rose et al. 2014; Stuecker et al. 2018) is polar amplification muted or eliminated.

Physical reasoning for the near-inevitability of polar amplification comes from the diffusive perspective. Preferential increase in MSE in the warm tropics relative to the cold poles with warming arises due to Clausius-Clapeyron scaling at constant relative humidity (Fig. 7a). This inherently leads to increased poleward AHT, preventing tropically amplified warming and contributing to polar-amplified warming. Viewed another way, partial homogenization of anomalous MSE by diffusion acts to preferentially increase the temperature of cold polar regions (Fig. 7b). Only when polar warming becomes strongly amplified is the MSE gradient sufficiently reduced that poleward AHT decreases into polar regions, as seen in the in the Northern Hemisphere of CMIP5 models (sections $3 b$ and $3 c$ ). This suggests that meridional AHT is a key driver of polar amplification, even while diagnostic warming contributions (Pithan and Mauritsen 2014; Goosse et al. 2018), taken at face value, appear to suggest otherwise.

Physical reasoning also comes from the energetic perspective. Driven by the same meridional patterns of radiative forcing, feedbacks, ocean heat uptake, Moist and Dry EBMs produce similar patterns of meridional AHT changes, but do so with very different patterns of warming (Figs. 4b,c, 6, and 8). This suggests a reinterpretation of the role of AHT in climate change: insofar as meridional AHT changes are determined by energetic constraints, the details of how AHT is related to surface temperature exert strong constraints on the pattern of warming. This is particularly true in regions of 
weak radiative response where energy balance must be regained primarily through anomalous AHT, but is a decent approximation at all latitudes provided that the atmosphere is sufficiently diffusive. A clean illustration of this principle is seen in polar regions under transient warming. Moist and Dry EBMs produce nearly identical reductions in poleward AHT (Fig. 6), yet they accomplish those changes in different ways: temperature diffusion requires a relatively small decrease in the temperature gradient, while MSE diffusion requires a large decrease in the temperature gradient (and thus strong polar amplification) in order to produce the required decrease in MSE gradient (Figs. 4b,c).

These findings also suggest a mechanism for why projections of warming are more uncertain in polar regions than in lower latitudes (e.g., Holland and Bitz 2003). Several recent studies (Rose et al. 2014; Kang et al. 2017; Park et al. 2018; Stuecker et al. 2018; Shaw and Tan 2018) find that forcing applied in the tropics results in a somewhat meridionally uniform warming while forcing applied in polar regions results in strongly polar-amplified warming. Likewise, Roe et al. (2015) and Bonan et al. (2018) demonstrate that radiative feedback uncertainty in the tropics results in meridionally uniform warming uncertainty while feedback uncertainty in polar regions results in warming uncertainty that is largely confined to the poles. An implication is that tropical warming uncertainty arises primarily from tropical processes (cloud feedbacks in particular), while polar warming uncertainty is driven by processes at all latitudes. This asymmetric behavior can be understood in the context of the framework presented here as a consequence of the greater efficiency with which poleward AHT changes are accomplished in the tropics than at the poles in a moist atmosphere: the change in MSE gradient necessary to realize a given change in AHT corresponds to a small modification to the temperature gradient in the tropics but a large modification to the temperature gradient in high latitudes (Fig. 7b). Latent energy transport thus fundamentally shapes 1 ) the climate's response to forcing, favoring polar amplification and 2) the predictability of climate change at different latitudes, favoring greater uncertainty in cold polar regions.

There are several qualifications to this interpretation, however. In the limit of small diffusivity or weak meridional variations in forcing, feedbacks, and ocean heat uptake, meridional AHT changes become sensitive to the details of how meridional AHT is related to surface temperature (i.e., on diffusivity value or on whether latent energy is accounted for; section $4 \mathrm{~d}$ ). We have also assumed that the behavior of AHT in the EBMs can be explored by varying the meridional pattern of feedbacks. This is a simplification given that feedback pattern is largely set by moist atmospheric processes and likely depends on the patterns of surface warming, ocean heat uptake, and AHT changes (e.g., Graversen and Wang 2009; Rose et al. 2014; Yoshimori et al. 2017; Feldl et al. 2017a; Singh et al. 2017; Po-Chedley et al. 2018). Moreover, ocean heat uptake has been prescribed within the EBMs, while its meridional pattern is thought to be set by regional ocean dynamics (Marshall et al. 2015; Armour et al. 2016), the degree to which the magnitude of regional ocean heat uptake depends on atmospheric processes is not known and should be explored in future work.

There also remain open questions regarding the role of atmospheric dynamics in meridional AHT. The results presented here suggest that atmospheric circulations must somehow act to satisfy energetic constraints on net AHT, but we have not identified the mechanism by which this is realized. A reasonable conjecture is that transient eddies act so efficiently that they are able to contribute whatever AHT is needed to make up the gap between the net AHT required and that provided by the other atmospheric circulation components (stationary eddy and meridional overturning). This would explain the blending of AHT by different components of the atmospheric circulation into the seamless meridional structure of net AHT. It would also provide justification for why the diffusive approximation for meridional AHT works so well. In this view, transient eddies set the effective diffusivity of the atmosphere, but we lack a theory for its exact value. The diffusive response found in our analyses is also reminiscent of a suggestion from Lorenz (1960) that such an adjustment mechanism might operate in a system that maximized the conversion of available potential energy to kinetic energy or equivalently, as has been subsequently shown, a system that maximized entropy production (e.g., Ozawa et al. 2003). It is also unclear whether the mechanisms for polar amplification identified here are consistent with those seen in observations over recent decades, which have been suggested to be associated with planetary wave dynamics (e.g., Lee 2014).

While $D_{m}$ is surely not meridionally uniform or constant over time (Shaw and Voigt 2016), the assumption that it is works surprisingly well. Yet, it is unclear why the diffusive approximation works so well within the deep tropics, where transient eddies contribute little to AHT, or how diffusing near-surface MSE provides a decent representation of transport over the whole atmospheric column. It seems that fruitful research directions would be the development of process-level understanding of how energetic constraints on meridional AHT become manifest through atmospheric 
dynamics and the examination of the limits of diffusive transport as an approximation to those dynamics.

Acknowledgments. We acknowledge support from National Science Foundation Award AGS-1752796 (KCA). This work benefited from insightful discussions with David Battisti, Cecilia Bitz, David Bonan, Nicole Feldl, Dennis Hartmann, Cristian Proistosescu, and Sarah Ragen. CMIP5 model output is available at the Earth System Grid Federation data portal (https:// esgf-node.llnl.gov/projects/cmip5/). ERA-Interim Reanalysis data are available at the ECMWF data portal (https://www.ecmwf.int/en/forecasts/datasets/archivedatasets/reanalysis-datasets/era-interim). CERES Energy Balance and Filled satellite radiation data are available at https://ceres.larc.nasa.gov/products.php? product5EBAF-TOA. We thank Timothy Merlis and an anonymous reviewer for their thoughtful comments and suggestions.

\section{APPENDIX A}

\section{Observations and Reanalyses}

We derive net TOA radiation fluxes from the Clouds and the Earth's Radiant Energy System (Wielicki et al. 1996) Energy Balance and Filled product (CERES EBAF; Loeb et al. 2009) version 4.0 from January 2001 to December 2016. We average the fields zonally over all years to define the climatology TOA radiation fluxes shown in Fig. 1b.

We use ERA-Interim (Dee et al. 2011) output from January 2001 to December 2016. We average the monthly near-surface air temperature zonally and over all years to define the climatological shown in Fig. 2. We use 6-hourly fields to calculate meridional energy fluxes for each month by employing Eq. (1) at each latitude and average the results over all years to define the climatological AHT shown in Fig. 1a. The meridional velocities $(v)$ and MSE $(h)$ are decomposed into meanmeridional and transient overturning, transient eddy, and stationary eddy components following Eq. (2). We account for conservation of mass in the meridional overturning circulation energy transport by removing the vertical average MSE, as in Marshall et al. (2014), rather than using a barotropic wind correction, as in Trenberth and Stepaniak (2003), because the resulting meridional overturning circulation energy transport has been shown to be more physically relevant on monthly time scales (Liang et al. 2018).

We calculate moist and dry components of meridional AHT, shown in Fig. 1d, in two different ways that give the same result: first, by use of Eq. (1) with MSE replaced with individual moist $(L q)$ and dry $\left(c_{p} T+g z\right)$ components, and second, by calculating the zonal-mean latent energy flux convergence from monthly precipitation minus evaporation fields; meridional latent heat transport is then derived by use of Eq. (4), and drystatic energy transport is then calculated as a residual from the net AHT calculated from TOA and surface energetic constraints.

We derive net surface heat flux fields for ERA-Interim as a residual between atmospheric energy convergence (calculated from the meridional energy fluxes above) and net TOA radiation fluxes from CERES EBAF. This provides a slightly different estimate of surface fluxes than derived directly from ERA-Interim, but ensures the same net meridional AHT in Figs. 1a, 1c, and 1d.

\section{APPENDIX B}

\section{Hadley Cell Parameterization of Tropical Moisture Transport}

Following Siler et al. (2018), we seek to partition the net AHT [Eq. (6)] into Hadley cell (HC) and eddy components: $F(x)=F_{\mathrm{HC}}(x)+F_{\text {eddy }}(x)$, where

$$
\begin{aligned}
F_{\mathrm{HC}}(x) & =w(x) F(x), \\
F_{\text {eddy }}(x) & =[1-w(x)] F(x),
\end{aligned}
$$

and $w$ is a Gaussian with width $\sigma=0.26\left(15^{\circ}\right)$ to represent the dominance of transient eddies in the extratropics and the Hadley cell within the tropics. We represent poleward AHT by the Hadley cell as

$$
F_{\mathrm{HC}}(x)=\psi(x) g(x),
$$

where $\psi(x)$ is the mass transport in each branch of the Hadley cell (with southward transport in the lower branch equal to northward transport in the upper branch by mass conservation); $g(x)$ is the gross moist stability, defined as the difference between MSE in the upper and lower branches at each latitude. Following Held (2001), we assume that MSE is relatively uniform, with value $h_{u}$, throughout the upper branch of the Hadley cell such that variations in $g(x)$ are primarily caused by meridional variations in near-surface MSE: $g(x) \approx h_{u}-h(x)$, where we set $h_{u}=1.07 \times h(0)$, or $7 \%$ above the near-surface MSE at the equator; this provides the best fit to tropical moisture transport and is a decent approximation of the observed atmospheric MSE profile at the equator (Siler et al. 2018).

Because $g(x)>0$ throughout the tropics, the Hadley cell parameterization produces downgradient (poleward) net transport of MSE. However, because the 


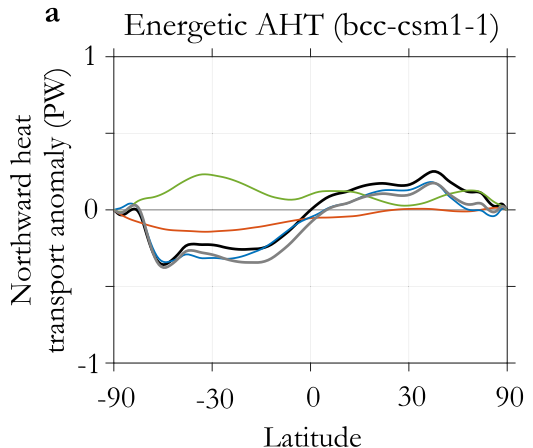

d Energetic AHT (CSIRO-Mk3-6-0)

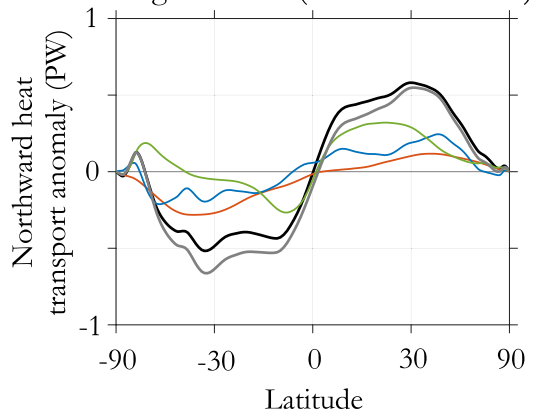

g Energetic AHT (IPSL-CM5A-LR)

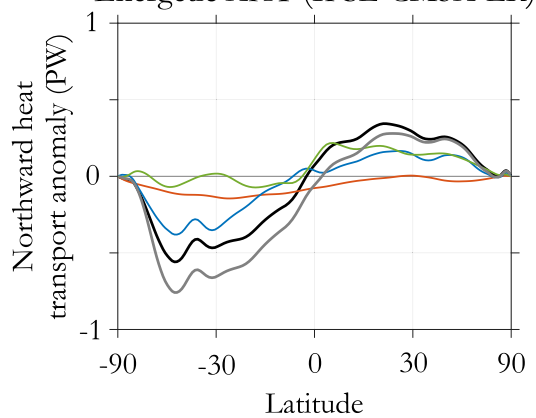

j Energetic AHT (MRI-CGCM3)

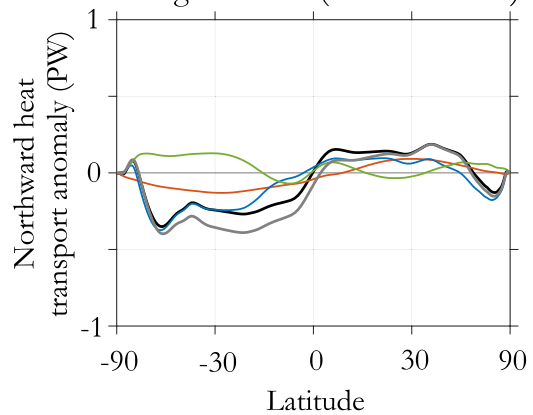

b

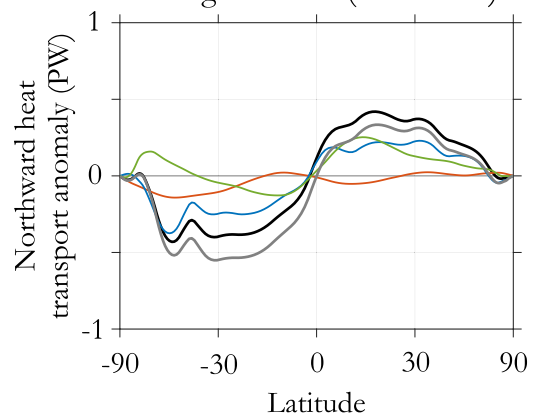

e Energetic AHT (GFDL-CM3)

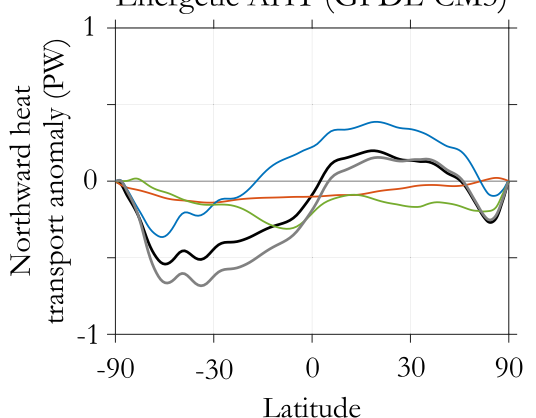

h Energetic AHT (MIROC5)

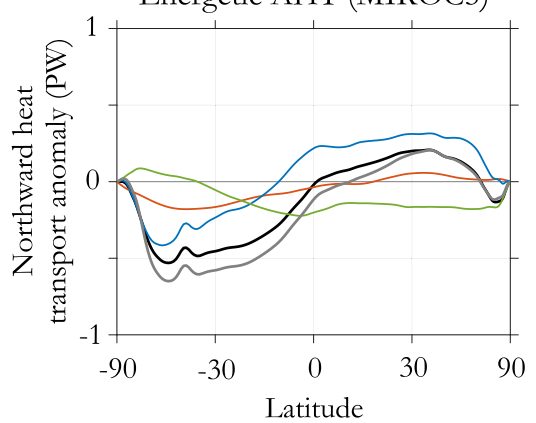

k Energetic AHT (NorESM1-M)

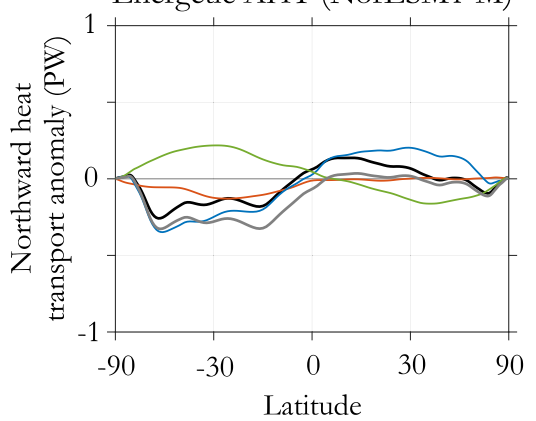

c

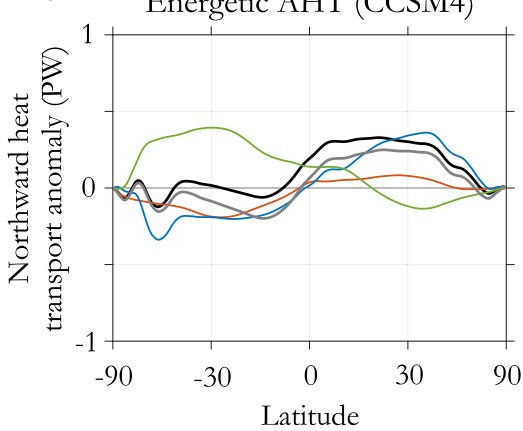

f

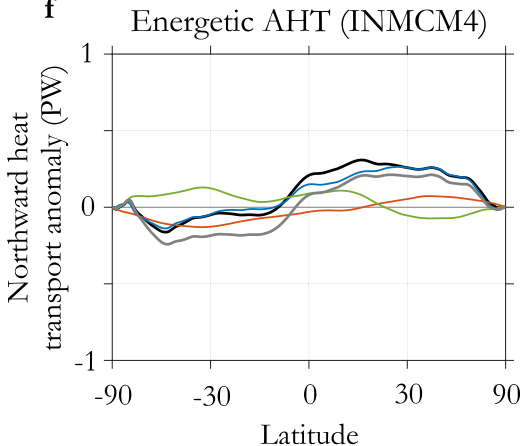

i Energetic AHT (MPI-ESM-LR)

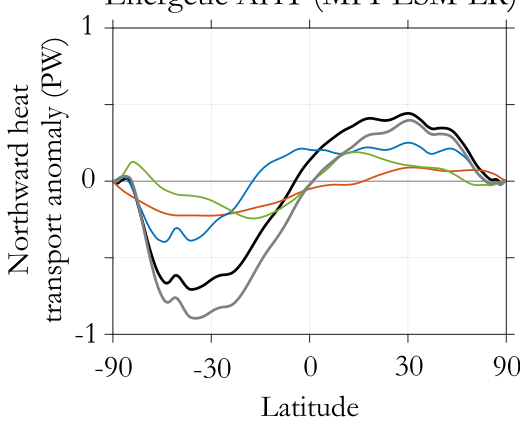

FIG. C1. Comparison of meridional atmospheric heat transport changes in individual CMIP5 GCMs and Moist EBMs (responses at year 100 following abrupt $\mathrm{CO}_{2}$ quadrupling). (a)-(k) Anomalous northward AHT in individual CMIP5 GCMs (black line) and Moist EBMs (gray line) driven with that GCM's radiative forcing, radiative feedbacks, and ocean heat uptake (section 4). Red line shows anomalous AHT implied by radiative forcing (same for GCM and EBM); blue line shows anomalous AHT implied by ocean heat uptake (same for GCM and EBM); green line shows anomalous AHT implied by radiative response (feedbacks) for GCMs. Ensemble means of GCMs and EBMs are shown in Figs. 3c and 6c, respectively. 
upper branch of the Hadley cell is essentially dry, moisture transport is confined to the lower branch and transported upgradient. We thus estimate latent energy transport by the Hadley cell according to

$$
\begin{aligned}
F_{\mathrm{HC}, q}(x) & =-\psi(x) L_{v} q(x) \\
& =\frac{w(x) F(x)}{1.07 \times h(0)-h(x)} L_{v} q(x),
\end{aligned}
$$

with dry-static energy transport equal to $F_{\mathrm{HC}, d}(x)=$ $F_{\mathrm{HC}}(x)-F_{\mathrm{HC}, q}(x)$.

\section{APPENDIX C}

\section{General Circulation Model Output}

We use monthly output from 11 CMIP5 GCMs that provide all necessary fields to calculate meridional AHT from all three perspectives for both the preindustrial control ("piControl") and abrupt $\mathrm{CO}_{2}$ quadrupling ("abrupt4xCO2") simulations: BCC_CSM1.1, CanESM2, CCSM4, CSIRO Mk3.6.0, GFDL-CM3, INM-CM4, IPSL-CM5A-LR, MIROC5, MPI-ESM-LR, MRICGCM3, and NorESM1-M. To account for model drift, we remove the linear trend of each model's preindustrial control simulation from all monthly variables prior to analysis; the trend is calculated over the 150 years following each model's branch time for the abrupt $\mathrm{CO}_{2}$ quadrupling simulation. Anomalies in abrupt $\mathrm{CO}_{2}$ quadrupling simulations are taken as averages over years $85-115$ relative to the 150 -yr average over the (drift corrected) control simulations. Anomalies in the CAM4 slab-ocean simulation are taken as differences between the model equilibrated at preindustrial $\mathrm{CO}_{2}$ levels and with $\mathrm{CO}_{2}$ doubled.

We calculate meridional AHT anomalies (Figs. 3a,c,d and $\mathrm{C} 1$ ) in two ways: first, by use of Eq. (8) applied to the residual between anomalous TOA radiation and net surface heat fluxes, and second, from a dynamical calculation of meridional energy fluxes according to Eqs. (1) and (2) at each latitude, as above, applied to anomalous velocity and MSE fields. However, because the fields are monthly, the transient eddy component is not accurate and is instead derived as a residual between the net AHT anomaly calculated from energetic constraints and the sum of AHT components associated with meanmeridional and transient overturning and stationary eddy fluxes.

Radiative forcing for the CMIP5 models, shown in Fig. 5a, is derived from $\mathrm{CO}_{2}$ quadrupling simulations with atmosphere-only versions of the models wherein sea surface temperatures and sea ice concentrations are fixed at preindustrial levels ("sstClim4xCO2"). CAM4's radiative forcing of $\mathrm{CO}_{2}$ doubling is assumed to be half that derived from $\mathrm{CO}_{2}$ quadrupling. Zonal-mean TOA radiation changes under increased $\mathrm{CO}_{2}$ averaged over the simulations are equated with the effective (or tropospheric-adjusted) radiative forcing $\left(R_{f}\right)$; we apply the standard correction to account for radiation associated with warming over land and sea ice by subtracting $1 \mathrm{~W} \mathrm{~m}^{-2} \mathrm{~K}^{-1}$ following Hansen et al. (2005). Zonalmean radiative feedbacks ( $\lambda$ shown in Fig. 5b) are calculated from the $\mathrm{CO}_{2}$ quadrupling (coupled CMIP5) or doubling (slab ocean CAM4) simulations by equating zonal-mean TOA radiation change with $\lambda(x) T^{\prime}(x)+$ $R_{f}(x)$. Net surface heat flux changes (ocean heat uptake shown in Fig. 5a) are calculated from net surface shortwave radiation, longwave radiation, and turbulent heat flux (sensible and latent) fields, as well as the latent heat associated with falling snow.

\section{REFERENCES}

Alexeev, V. A., and C. H. Jackson, 2013: Polar amplification: Is atmospheric heat transport important? Climate Dyn., 41, 533547, https://doi.org/10.1007/s00382-012-1601-z.

Armour, K. C., C. M. Bitz, and G. H. Roe, 2013: Time-varying climate sensitivity from regional feedbacks. J. Climate, 26, 4518-4534, https://doi.org/10.1175/JCLI-D-12-00544.1.

, J. Marshall, J. Scott, A. Donohoe, and E. R. Newsom, 2016: Southern Ocean warming delayed by circumpolar upwelling and equatorward transport. Nat. Geosci., 9, 549-554, https:// doi.org/10.1038/ngeo2731.

Barnes, E. A., and L. M. Polvani, 2013: Response of the midlatitude jets and of their variability to increased greenhouse gases in the CMIP5 models. J. Climate, 26, 7117-7135, https://doi.org/ 10.1175/JCLI-D-12-00536.1.

Bjerknes, J., 1964: Atlantic air-sea interaction. Advances in Geophysics, Vol.10, Academic Press, 1-82, https://doi.org/10.1016/ S0065-2687(08)60005-9.

Bonan, D. B., K. C. Armour, G. H. Roe, N. Siler, and N. Feldl, 2018: Sources of uncertainty in the meridional pattern of climate change. Geophys. Res. Lett., 45, 9131-9140, https:// doi.org/10.1029/2018GL079429.

Budyko, M. I., 1969: The effect of solar radiation variations on the climate of the Earth. Tellus, 21, 611-619, https://doi.org/ 10.3402/tellusa.v21i5.10109.

Ceppi, P., and D. L. Hartmann, 2016: Clouds and the atmospheric circulation response to warming. J. Climate, 29, 783-799, https://doi.org/10.1175/JCLI-D-15-0394.1.

- and T. G. Shepherd, 2017: Contributions of climate feedbacks to changes in atmospheric circulation. J. Climate, 30, 90979118, https://doi.org/10.1175/JCLI-D-17-0189.1.

, M. D. Zelinka, and D. L. Hartmann, 2014: The response of the Southern Hemispheric eddy-driven jet to future changes in shortwave radiation in CMIP5. Geophys. Res. Lett., 41, 3244 3250, https://doi.org/10.1002/2014GL060043.

Crook, J. A., P. M. Forster, and N. Stuber, 2011: Spatial patterns of modeled climate feedback and contributions to temperature response and polar amplification. J. Climate, 24, 3575-3592, https://doi.org/10.1175/2011JCLI3863.1. 
Dee, D. P., and Coauthors, 2011: The ERA-Interim reanalysis: Configuration and performance of the data assimilation system. Quart. J. Roy. Meteor. Soc., 137, 553-597, https://doi.org/ 10.1002/qj.828.

Donohoe, A., and D. S. Battisti, 2012: What determines meridional heat transport in climate models? J. Climate, 25, 3832-3850, https://doi.org/10.1175/JCLI-D-11-00257.1.

-, J. Marshall, D. Ferreira, and D. McGee, 2013: The relationship between ITCZ location and cross-equatorial atmospheric heat transport: From the seasonal cycle to the Last Glacial Maximum. J. Climate, 26, 3597-3618, https://doi.org/10.1175/ JCLI-D-12-00467.1.

,,--- K. C. Armour, and D. McGee, 2014: The interannual variability of tropical precipitation and interhemispheric energy transport. J. Climate, 27, 3377-3392, https://doi.org/10.1175/JCLI-D-13-00499.1.

Enderton, D., and J. Marshall, 2009: Explorations of atmosphereocean-ice climates on an aquaplanet and their meridional energy transports. J. Atmos. Sci., 66, 1593-1611, https:// doi.org/10.1175/2008JAS2680.1.

Fasullo, J. T., and K. E. Trenberth, 2008: The annual cycle of the energy budget. Part II: Meridional structures and poleward transports. J. Climate, 21, 2313-2325, https://doi.org/10.1175/ 2007JCLI1936.1.

Feldl, N., and S. Bordoni, 2016: Characterizing the Hadley circulation response through regional climate feedbacks. $J$. Climate, 29, 613-622, https://doi.org/10.1175/JCLI-D-150424.1.

— , and G. H. Roe, 2013a: The nonlinear and nonlocal nature of climate feedbacks. J. Climate, 26, 8289-8304, https://doi.org/ 10.1175/JCLI-D-12-00631.1.

$\longrightarrow$, and - 2013b: Four perspectives on climate feedbacks. Geophys. Res. Lett., 40, 4007-4011, https://doi.org/10.1002/ grl.50711.

— D. M. W. Frierson, and G. H. Roe, 2014: The influence of regional feedbacks on circulation sensitivity. Geophys. Res. Lett., 41, 2212-2220, https://doi.org/10.1002/2014GL059336.

_ B. B. T. Anderson, and S. Bordoni, 2017a: Atmospheric eddies mediate lapse rate feedback and Arctic amplification. J. Climate, 30, 9213-9224, https://doi.org/10.1175/JCLI-D-16-0706.1.

— S. Bordoni, and T. M. Merlis, 2017b: Coupled high-latitude climate feedbacks and their impact on atmospheric heat transport. J. Climate, 30, 189-201, https://doi.org/10.1175/ JCLI-D-16-0324.1.

Flannery, B. P., 1984: Energy balance models incorporating transport of thermal and latent energy. J. Atmos. Sci., 41, 414-421, https://doi.org/10.1175/1520-0469(1984)041<0414: EBMITO $>2.0 . \mathrm{CO} ; 2$

Frierson, D. M. W., and Y.-T. Hwang, 2012: Extratropical influence on ITCZ shifts in slab ocean simulations of global warming. J. Climate, 25, 720-733, https://doi.org/10.1175/JCLI-D-1100116.1.

— I. M. Held, and P. Zurita-Gotor, 2007: A gray-radiation aquaplanet moist GCM. Part II: Energy transports in altered climates. J. Atmos. Sci., 64, 1680-1693, https://doi.org/10.1175/ JAS3913.1.

— , and Coauthors, 2013: Contribution of ocean overturning circulation to tropical rainfall peak in the Northern Hemisphere. Nat. Geosci., 6, 940-944, https://doi.org/10.1038/ ngeo1987.

Goosse, H., and Coauthors, 2018: Quantifying climate feedbacks in polar regions. Nat. Commun., 9, 1919, https://doi.org/10.1038/ s41467-018-04173-0.
Graversen, R. G., and M. Wang, 2009: Polar amplification in a coupled climate model with locked albedo. Climate Dyn., 33, 629-643, https://doi.org/10.1007/s00382-009-0535-6.

— , and M. Burtu, 2016: Arctic amplification enhanced by latent energy transport of atmospheric planetary waves. Quart. J. Roy. Meteor. Soc., 142, 2046-2054, https://doi.org/10.1002/ qj.2802.

Hansen, J., and Coauthors, 2005: Efficacy of climate forcings. J. Geophys. Res., 110, D18104, https://doi.org/10.1029/ 2005JD005776.

Hartmann, D. L., 2016: Global Physical Climatology. 2nd ed. Elsevier, $485 \mathrm{pp}$.

Held, I. M., 1999: The macroturbulence of the troposphere. Tellus, 51B, 59-70, https://doi.org/10.3402/tellusb.v51i1.16260. , 2001: The partitioning of the poleward energy transport between the tropical ocean and atmosphere. J. Atmos. Sci., $\mathbf{5 8}$, 943-948, https://doi.org/10.1175/1520-0469(2001)058<0943: TPOTPE $>2.0 . \mathrm{CO} ; 2$.

— cycle to global warming. J. Climate, 19, 5686-5699, https:// doi.org/10.1175/JCLI3990.1.

Holland, M. M., and C. M. Bitz, 2003: Polar amplification of climate change in coupled models. Climate Dyn., 21, 221-232, https:// doi.org/10.1007/s00382-003-0332-6.

Holton, J. R., and G. J. Hakim, 2013: An Introduction to Dynamic Meteorology. Academic Press, 532 pp.

Huang, P., S.-P. Xie, K. Hu, G. Huang, and R. Huang, 2013: Patterns of the seasonal response of tropical rainfall to global warming. Nat. Geosci., 6, 357-361, https://doi.org/10.1038/ ngeo1792.

Huang, Y., and M. Zhang, 2014: The implication of radiative forcing and feedback for meridional energy transport. Geophys. Res. Lett., 41, 1665-1672, https://doi.org/10.1002/ 2013GL059079.

Hwang, Y.-T., and D. M. W. Frierson, 2010: Increasing atmospheric poleward energy transport with global warming. Geophys. Res. Lett., 37, L24807, https://doi.org/10.1029/2010GL045440.

—, and —, 2013: Link between the double-Intertropical Convergence Zone problem and cloud bias over Southern Ocean. Proc. Natl. Acad. Sci. USA, 110, 4935-4940, https:// doi.org/10.1073/pnas.1213302110.

_,$\ldots$, and J. E. Kay, 2011: Coupling between Arctic feedbacks and changes in poleward energy transport. Geophys. Res. Lett., 38, L17704, https://doi.org/10.1029/ 2011 GL048546.

Kang, S. M., I. M. Held, D. M. W. Frierson, and M. Zhao, 2008: The response of the ITCZ to extratropical forcing: Idealized slabocean experiments with a GCM. J. Climate, 21, 3521-3532, https://doi.org/10.1175/2007JCLI2146.1.

— , K. Park, F.-F. Jin, and M. F. Stuecker, 2017: Common warming pattern emerges irrespective of forcing location. J. Adv. Model. Earth Syst., 9, 2413-2424, https://doi.org/ 10.1002/2017MS001083.

Kay, J. E., M. M. Holland, C. M. Bitz, E. Blanchard-Wrigglesworth, A. Gettleman, A. Conley, and D. Bailey, 2012: The influence of local feedbacks and northward heat transport on the equilibrium Arctic climate response to increased greenhouse gas forcing. J. Climate, 25, 5433-5450, https://doi.org/10.1175/ JCLI-D-11-00622.1.

, and Coauthors, 2016: Global climate impacts of fixing the Southern Ocean shortwave radiation bias in the Community Earth System Model. J. Climate, 29, 4617-4636, https://doi.org/ 10.1175/JCLI-D-15-0358.1. 
Lee, S., 2014: A theory for polar amplification from a general circulation perspective. Asia-Pac. J. Atmos. Sci., 50, 31-43, https://doi.org/10.1007/s13143-014-0024-7.

Liang, M., A. Czaja, R. Graversen, and R. Tailleux, 2018: Poleward energy transport: Is the standard definition physically relevant at all time scales? Climate Dyn., 50, 1785-1797, https://doi.org/ 10.1007/s00382-017-3722-x.

Liu, C., and E. A. Barnes, 2015: Extreme moisture transport into the Arctic linked to Rossby wave breaking. J. Geophys. Res. Atmos., 120, 3774-3788, https://doi.org/10.1002/2014JD022796.

Liu, X., D. S. Battisti, and G. H. Roe, 2017: The effect of cloud cover on the meridional heat transport: Lessons from variable rotation experiments. J. Climate, 30, 7465-7479, https:// doi.org/10.1175/JCLI-D-16-0745.1.

Liu, Z., H. Yang, C. He, and Y. Zhao, 2016: A theory for Bjerknes compensation: The tole of climate feedback. J. Climate, 29, 191-208, https://doi.org/10.1175/JCLI-D-15-0227.1.

Loeb, N. G., B. A. Wielicki, D. R. Doelling, G. L. Smith, D. F. Keyes, S. Kato, N. Manalo-Smith, and T. Wong, 2009: Toward optimal closure of the earth's top-of-atmosphere radiation budget. J. Climate, 22, 748-766, https://doi.org/10.1175/2008JCLI2637.1.

Lorenz, E. N., 1960: Generation of available potential energy and the intensity of the general circulation. Dynamics of Climate, R. L. Pfeffer, Ed., Pergamon, 86-92.

Lu, J., G. A. Vecchi, and T. Reichler, 2007: Expansion of the Hadley cell under global warming. Geophys. Res. Lett., 34, L06805, https://doi.org/10.1029/2006GL028443.

Marshall, J., A. Donohoe, D. Ferreira, and D. McGee, 2014: The ocean's role in setting the mean position of the Inter-Tropical Convergence Zone. Climate Dyn., 42, 1967-1979, https:// doi.org/10.1007/s00382-013-1767-z.

- , J. Scott, K. C. Armour, J.-M. Campin, M. Kelley, and A. Romanou, 2015: The ocean's role in the transient response of climate to abrupt greenhouse gas forcing. Climate Dyn., $\mathbf{4 4}$, 2287-2299, https://doi.org/10.1007/s00382-014-2308-0.

Mbengue, C., and T. Schneider, 2017: Storm-track shifts under climate change: Toward a mechanistic understanding using baroclinic mean available potential energy. J. Atmos. Sci., 74 93-110, https://doi.org/10.1175/JAS-D-15-0267.1.

—, and —, 2018: Linking Hadley circulation and storm tracks in a conceptual model of the atmospheric energy balance. J. Atmos. Sci., 75, 841-856, https://doi.org/10.1175/JAS-D-17-0098.1.

McFarlane, A. A., and D. M. W. Frierson, 2017: The role of ocean fluxes and radiative forcings in determining tropical rainfall shifts in RCP8.5 simulations. Geophys. Res. Lett., 44, 86568664, https://doi.org/10.1002/2017GL074473.

Merlis, T. M., 2014: Interacting components of the top-ofatmosphere energy balance affect changes in regional surface temperature. Geophys. Res. Lett., 41, 7291-7297, https:// doi.org/10.1002/2014GL061700.

_ 2015: Direct weakening of tropical circulations from masked $\mathrm{CO}_{2}$ radiative forcing. Proc. Natl. Acad. Sci. USA, 112, 13167 13171, https://doi.org/10.1073/pnas.1508268112.

_- and M. Henry, 2018: Simple estimates of polar amplification in moist diffusive energy balance models. J. Climate, 31, 58115824, https://doi.org/10.1175/JCLI-D-17-0578.1.

Neale, R. B., and Coauthors, 2013: The mean climate of the Community Atmosphere Model (CAM4) in forced SST and fully coupled experiments. J. Climate, 26, 5150-5168, https:// doi.org/10.1175/JCLI-D-12-00236.1.

Neelin, J. D., C. Chou, and H. Su, 2003: Tropical drought regions in global warming and El Niño teleconnections. Geophys. Res. Lett., 30, 2275, https://doi.org/10.1029/2003GL018625.
North, G. R., 1975: Theory of energy-balance models. J. Atmos. Sci., 32, 2033-2043, https://doi.org/10.1175/1520-0469(1975) 032<2033:TOEBCM>2.0.CO;2.

_ 1981: Energy balance climate models. Rev. Geophys. Space Phys., 19, 91-121, https://doi.org/10.1029/RG019i001p00091.

Ozawa, H., A. Ohmura, R. D. Lorenz, and T. Pujol, 2003: The second law of thermodynamics and the global climate system: A review of the maximum entropy production principle. Rev. Geophys., 41, 1018-1041, https://doi.org/10.1029/ 2002RG000113.

Park, K., S. M. Kang, D. Kim, M. F. Stuecker, and F.-F. Jin, 2018: Contrasting local and remote impacts of surface heating on polar warming and amplification. J. Climate, 31, 3155-3166, https://doi.org/10.1175/JCLI-D-17-0600.1.

Pithan, F., and T. Mauritsen, 2014: Arctic amplification dominated by temperature feedbacks in contemporary climate models. Nat. Climate Change, 7, 181-184, https://doi.org/10.1038/ ngeo2071.

Po-Chedley, S., K. C. Armour, C. M. Bitz, M. D. Zelinka, B. D. Santer, and Q. Fu, 2018: Sources of intermodel spread in the lapse rate and water vapor feedbacks. J. Climate, 31, 31873206, https://doi.org/10.1175/JCLI-D-17-0674.1.

Roe, G. H., N. Feldl, K. C. Armour, Y.-T. Hwang, and D. M. W. Frierson, 2015: The remote impacts of climate feedbacks on regional climate predictability. Nat. Geosci., 8, 135-139, https://doi.org/10.1038/ngeo2346.

Rose, B. E. J., and D. Ferreira, 2013: Ocean heat transport and water vapor greenhouse in a warm equable climate: A new look at the low gradient paradox. J. Climate, 26, 2117-2136, https://doi.org/10.1175/JCLI-D-11-00547.1.

, K. C. Armour, D. S. Battisti, N. Feldl, and D. D. B. Koll, 2014: The dependence of transient climate sensitivity and radiative feedbacks on the spatial pattern of ocean heat uptake. Geophys. Res. Lett., 41, 1071-1078, https://doi.org/10.1002/2013GL058955.

Sellers, W. D., 1969: A global climatic model based on the energy balance of the earth-atmosphere system. J. Appl. Meteor., 8, 392-400, https://doi.org/10.1175/1520-0450(1969)008<0392: AGCMBO $>2.0 . \mathrm{CO} ; 2$

Shaw, T. A., and A. Voigt, 2016: What can moist thermodynamics tell us about circulation shifts in response to uniform warming? Geophys. Res. Lett., 43, 4566-4575, https://doi.org/ 10.1002/2016GL068712.

- and Z. Tan, 2018: Testing latitudinally dependent explanations of the circulation response to increased $\mathrm{CO}_{2}$ using aquaplanet models. Geophys. Res. Lett., 45, 9861-9869, https:// doi.org/10.1029/2018GL078974.

Siler, N., G. H. Roe, and K. C. Armour, 2018: Insights into the zonal-mean response of the hydrologic cycle to global warming from a diffusive energy balance model. J. Climate, 31, 7481-7493, https://doi.org/10.1175/JCLI-D-18-0081.1.

Singh, H. A., P. J. Rasch, and B. E. J. Rose, 2017: Increased ocean heat convergence into the high latitudes with $\mathrm{CO}_{2}$ doubling enhances polar-amplified warming. Geophys. Res. Lett., 44, 10 583-10 591, https://doi.org/10.1002/2017GL074561.

Stephens, G. L., D. O'Brien, P. J. Webster, P. Pilewski, S. Kato, and J.-L. Li, 2015: The albedo of Earth. Rev. Geophys., 53, 141163, https://doi.org/10.1002/2014RG000449.

Stone, P. H., 1978: Constraints on dynamical transports of energy on a spherical planet. Dyn. Atmos. Oceans, 2, 123-139, https:// doi.org/10.1016/0377-0265(78)90006-4.

Stuecker, M., and Coauthors, 2018: Polar amplification dominated by local forcing and feedbacks. Nat. Climate Change, 8, 10761081, https://doi.org/10.1038/s41558-018-0339-y. 
Taylor, K. E., R. J. Stouffer, and G. A. Meehl, 2012: An overview of CMIP5 and the experiment design. Bull. Amer. Meteor. Soc., 93, 485-498, https://doi.org/10.1175/BAMS-D-11-00094.1.

Trenberth, K. E., and J. M. Caron, 2001: Estimates of meridional atmosphere and ocean heat transports. J. Climate, 14, 3433-3443, https://doi.org/10.1175/1520-0442(2001)014<3433: EOMAAO $>2.0 . \mathrm{CO} ; 2$.

— energy transports and implications for the Hadley circulation. J. Climate, 16, 3706-3722, https://doi.org/10.1175/15200442(2003)016<3706:spaeta $>2.0$. co;2.

Voigt, A., and T. A. Shaw, 2015: Circulation response to warming shaped by radiative changes of clouds and water vapour. Nat. Geosci., 8, 102-106, https://doi.org/10.1038/ngeo2345.

- , and,- 2016 : Impact of regional atmospheric cloud radiative changes on shifts of the extratropical jet stream in response to global warming. J. Climate, 29, 8399-8421, https:// doi.org/10.1175/JCLI-D-16-0140.1.

, B. Stevens, J. Bader, and T. Mauritsen, 2013: The observed hemispheric symmetry in reflected shortwave irradiance. J. Climate, 26, 468-477, https://doi.org/10.1175/JCLI-D-12-00132.1.

Wagner, T. J. W., and I. Eisenman, 2015: How climate model complexity influences sea ice stability. J. Climate, 28, 39984014, https://doi.org/10.1175/JCLI-D-14-00654.1.

Watt-Meyer, O., and D. M. W. Frierson, 2017: Local and remote impacts of atmospheric cloud radiative effects onto the eddy-driven jet. Geophys. Res. Lett., 44, 10 036-10 044, https:// doi.org/10.1002/2017GL074901.

Wielicki, B., B. Barkstrom, E. Harrison, R. Lee, G. Smith, and J. Cooper, 1996: Clouds and the Earth's Radiant Energy System (CERES): An Earth observing system experiment. Bull. Amer. Meteor. Soc., 77, 853-868, https://doi.org/10.1175/ 1520-0477(1996)077<0853:CATERE >2.0.CO;2.

Wu, Y., M. Ting, R. Seager, H. Huang, and M. Cane, 2011: Changes in storm tracks and energy transports in a warmer climate simulated by the GFDL CM2.1 model. Climate Dyn., 37, 5372, https://doi.org/10.1007/s00382-010-0776-4.

Yin, J. H., 2005: A consistent poleward shift of the storm tracks in simulations of 21st century climate. Geophys. Res. Lett., 32, L18701, https://doi.org/10.1029/2005GL023684.

Yoshimori, M., A. Abe-Ouchi, and A. Laine, 2017: The role of atmospheric heat transport and regional feedbacks in the Arctic warming at equilibrium. Climate Dyn., 49, 3457-3472, https://doi.org/10.1007/s00382-017-3523-2.

Zelinka, M. D., and D. L. Hartmann, 2012: Climate feedbacks and their implications for poleward energy flux changes in a warming climate. J. Climate, 25, 608-624, https://doi.org/ 10.1175/JCLI-D-11-00096.1.

Zhang, R., and Coauthors, 2018: Local radiative feedbacks over the Arctic based on observed short-term climate variations. Geophys. Res. Lett., 45, 5761-5770, https://doi.org/10.1029/ 2018 GL077852. 